ARTICLE

\title{
Revealing thermally-activated nucleation pathways of diffusionless solid-to-solid transition
}

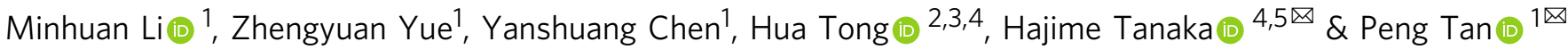

Solid-to-solid transitions usually occur via athermal nucleation pathways on pre-existing defects due to immense strain energy. However, the extent to which athermal nucleation persists under low strain energy comparable to the interface energy, and whether thermallyactivated nucleation is still possible are mostly unknown. To address these questions, the microscopic observation of the transformation dynamics is a prerequisite. Using a charged colloidal system that allows the triggering of an fcc-to-bcc transition while enabling in-situ single-particle-level observation, we experimentally find both athermal and thermallyactivated pathways controlled by the softness of the parent crystal. In particular, we reveal three new transition pathways: ingrain homogeneous nucleation driven by spontaneous dislocation generation, heterogeneous nucleation assisted by premelting grain boundaries, and wall-assisted growth. Our findings reveal the physical principles behind the systemdependent pathway selection and shed light on the control of solid-to-solid transitions through the parent phase's softness and defect landscape.

\footnotetext{
${ }^{1}$ State Key Laboratory of Surface Physics and Department of Physics, Fudan University, Shanghai, China. ${ }^{2}$ School of Physics and Astronomy, Shanghai Jiao Tong University, Shanghai, China. ${ }^{3}$ Department of Physics, University of Science and Technology of China, Hefei, China. ${ }^{4}$ Department of Fundamental Engineering, Institute of Industrial Science, University of Tokyo, Tokyo, Japan. ${ }^{5}$ Research Center for Advanced Science and Technology, University of Tokyo,

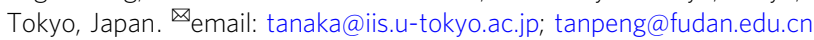


D iffusionless solid-to-solid transitions (martensitic transition) have been widely observed in both hard materials, such as metals, alloys, and ceramics ${ }^{1-10}$, and soft-matter systems, such as protein solutions and colloidal suspensions ${ }^{11-14}$. Despite the variety of these systems with various softness and symmetry ${ }^{4-14}$, one common intriguing feature is the formation of rich microstructures via complex nucleation and growth pathways. Nuclei formation has so far been believed to be athermal because immense strain energy is required to overcome a high barrier, which is the case for the steel's martensitic transition. This means that the process must be initiated from defectstabilised nuclei already existing in the parent solid well above the transformation temperature. The subsequent growth of the product solid is then governed by a delicate balance between elastic, interfacial, and chemical energies ${ }^{1}$.

However, such a scenario may no longer apply to soft materials in which the lattice is easy to deform because thermal activation cannot be neglected anymore. Nonetheless, to what extent the athermal nucleation persists as the strain energy reduces and whether there is a new realm of nucleation behaviours, e.g. thermally activated nucleation and interface energy-dominated pathways, remains mostly unknown. To fully understand the kinetics of solid-to-solid transformation, it is critical to elucidate softnessdependent nucleation behaviours. Understanding these processes will have a considerable impact on the microstructure control of numerous soft materials.

Martensitic transitions have been experimentally observed through X-ray scattering ${ }^{15}$, acoustic emission ${ }^{16}$, transmission electron microscopy ${ }^{17}$ and calorimetry ${ }^{18}$. However, these techniques can rarely access the single-particle-level nucleation kinetics of martensitic transitions. Fortunately, as an important class of soft materials, colloidal suspensions are suitable for real-space threedimensional (3D) microscopy observations. The controllability of the size, shape, and interparticle interaction of colloidal particles has provided deep microscopic insights into various phase transitions, including crystallisation ${ }^{19-22}$, melting ${ }^{23,24}$, glass transition ${ }^{25}$ as well as solid-to-solid transitions. Concerning martensitic transitions in colloidal crystals, many studies have been performed by applying an electric field ${ }^{12,26}$, changing the particle shape, confinement or volume fractions ${ }^{14,27-32}$, shear ${ }^{33,34}$ and adjusting the DNA grafting ${ }^{13,35}$. These studies have confirmed the diffusionless nature of the transition, as in hard materials.

However, even with colloidal particles, it has been quite challenging to experimentally access the microscopic kinetics in the nucleation stage due to the difficulty in a non-perturbative initiation of the transition while performing in-situ 3D confocal microscopy observations. Accordingly, the following two critical issues concerning the martensitic transition kinetics in soft materials have so far remained largely unexplored: (1) To what extent does the soft nature of materials affect the nucleation kinetics? (2) How does the system choose appropriate nucleation pathways to overcome the free-energy barrier? Here, we address these fundamental questions experimentally with a unique ion-exchange method that can initiate an fcc-to-bcc transition in a non-perturbative manner. We find both athermal and thermally activated pathways controlled by the softness of the parent crystal. In particular, we reveal three new transition pathways: the in-grain homogeneous nucleation driven by spontaneous dislocation generation, the heterogeneous nucleation assisted by premelting grain boundaries, and the wall-assisted growth.

\section{Results}

Experiments and methods. To access the kinetic process of solidsolid transition microscopically, we have designed a new experimental setup using a charged colloidal system, which allows us to control the parent fcc crystal's softness and probe its influence on the kinetics of fcc-to-bcc transitions at a single-particle-level resolution. Specifically, we use charged PMMA (polymethyl methacrylate) colloidal particles (diameter $\sigma \sim 1.8 \mu \mathrm{m}$, dyed with nitrobenzoxadiazole) suspended in a density- and refractive index-matched solvent. The interparticle interaction can be effectively described by the hard-core repulsive Yukawa potential: $u(r)=\alpha \exp [-\kappa \sigma(r / \sigma-1)] /(r / \sigma)$ for $r>\sigma$, with $1 / \kappa$ being the Debye screening length in the suspension and $\alpha$ being a parameter of the interaction strength. $1 / \kappa$ and $\alpha$ can be adjusted by the ionic AOT (sodium di-2-ethylhexyl sulfosuccinate) surfactant concentration, $C_{\mathrm{AOT}}{ }^{36-38}$. We employ a special ion-exchange experimental protocol (see Fig. 1a) that allows us to change the interparticle interaction, $u(r)$, quickly by adjusting $C_{\mathrm{AOT}}$ in the reservoir. We trigger the fcc-to-bcc transition at various $C_{\mathrm{AOT}}$ and observe the kinetics in-situ using a Leica SP8 fast confocal microscope. This method enables us to follow the microscopic kinetics of martensitic transitions with a single-particle-level resolution from the very beginning (see 'Methods' on the details of experiments).

We use two structural order parameters, which are coarsegrained bond orientational order parameters, $W_{6}$ and $Q_{6}$, to quantify the single-particle-level local structures (see Methods on their definitions). $W_{6}$ is used to distinguish fcc and bcc by its sign ( $W_{6} \geq 0$ for bcc and $W_{6}<0$ for fcc), whereas $Q_{6}$ quantifies the degree of crystalline order of the solid (particles with $Q_{6}<0.35$ ) are regarded as defective structures, which mainly consist of highly distorted (defective) solids with a small amount of liquidlike $\left(Q_{6}<0.25\right)$ particles $^{21,22,39-41}$ (Fig. 2).

Softness-dependent nucleation behaviours. In general, the nucleation barrier for the formation of the nucleus of the product phase can be written as ${ }^{1,31}$ :

$$
\Delta G=-V \rho \Delta \mu+A \gamma+E_{\text {strain }}-E_{\text {defect }},
$$

where $V$ is the nucleus volume of the product phase, $\rho$ is the particle number density of the product nuclei, $\Delta \mu(>0)$ is the chemical-potential difference between the parent and product phases, $A$ is the nucleus surface area, $\gamma$ is the surface tension, $E_{\text {strain }}$ is the extra misfit strain energy induced by the volume difference between the two phases that depends on the elastic modulus of the parent phase, and $E_{\text {defect }}$ is the energy of preexisting defects per volume $V$. The first term on the right-hand side is a driving force of the transition. The second and third terms represent the surface- and strain-energy costs of the product phase's nucleation. In hard materials, $E_{\text {strain }}$ is usually quite large, and the nucleation barrier is much larger than the thermal energy, $k_{B} T$. Accordingly, internal or applied stress is the major controlling factor of nuclei generation. We refer to this case as athermal nucleation. In such a case, pre-existing defects promote the nucleation because defective structures have low local modulus and significantly reduce $E_{\text {strain }}$. It was phenomenologically shown for athermal nucleation behaviours in steels that nuclei stabilised by stresses already pre-exist in the parent phase, and their growth is governed by the balance between $E_{\text {strain }}, A \gamma$, and $\Delta \mu$. In contrast, the much smaller $E_{\text {strain }}$ in soft materials than hard materials can provide other nucleation pathways to reduce the nucleation barrier, depending on the condition. They include spontaneous nucleation with and without applied stresses and pathways controlled by the interface energy $A \gamma$. Phenomenologically, thermally driven nucleation could happen with or without applied stresses. For example, a previous study of colloidal thin films revealed a nucleation behaviour triggered by the internal fluid flow, which can be regarded as stress-induced spontaneous nucleation ${ }^{14}$. 
a

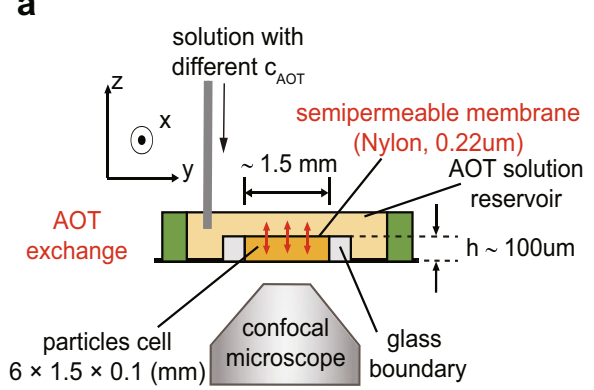

d

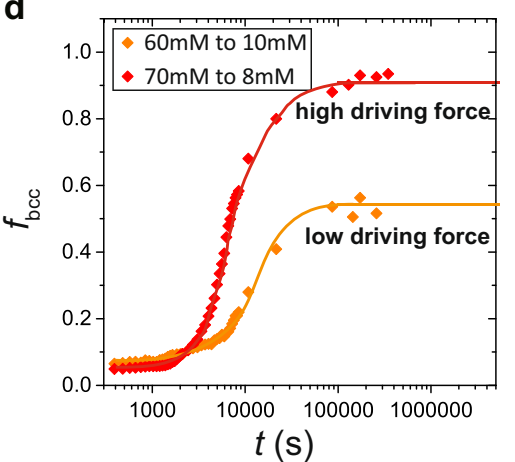

b

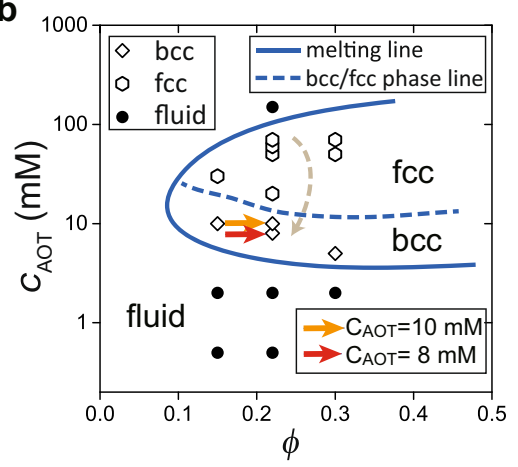

c

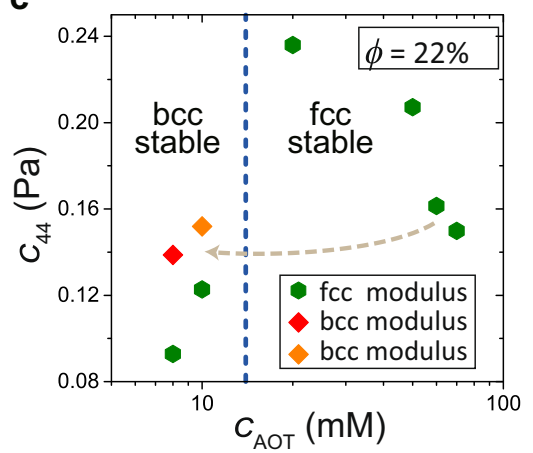

e

\begin{tabular}{|c|c|c|c|}
\hline Pathway & $\begin{array}{c}\text { Low Driven Force } \\
(\mathbf{6 0 m M} \text { to } 10 \mathrm{mM}, \\
\mathbf{C}_{44}^{\text {fcc }} \mathbf{0 . 1 2 P a )}\end{array}$ & $\begin{array}{c}\text { High Driven Force } \\
(\mathbf{7 0 m M} \text { to } 8 \mathrm{mM}, \\
\left.\mathbf{C}_{44}^{\text {fcc }} \sim \mathbf{0 . 0 9 P a}\right)\end{array}$ & $\begin{array}{c}\text { Orientation } \\
\text { Relationship }\end{array}$ \\
\hline $\begin{array}{c}\text { Inside-grain } \\
\text { Nucleation }\end{array}$ & $\begin{array}{c}\text { Pre-existing nuclei, } \\
\text { athermal }\end{array}$ & $\begin{array}{l}\text { Thermally-activated, } \\
\text { contribute } \sim 30 \% \text { bcc }\end{array}$ & $\begin{array}{c}\text { NW relation, } \\
\text { KS relation }\end{array}$ \\
\hline $\begin{array}{c}\text { Grain Boundary } \\
\text { Nucleation }\end{array}$ & $\begin{array}{c}\text { Pre-existing nuclei, } \\
\text { athermal }\end{array}$ & $\begin{array}{l}\text { Thermally-activated, } \\
\text { contribute } \sim 70 \% \text { bcc }\end{array}$ & NW relation \\
\hline $\begin{array}{c}\text { Wall } \\
\text { Nucleation }\end{array}$ & $\begin{array}{c}\text { Pre-existing nuclei, } \\
\text { athermal }\end{array}$ & No & NW relation \\
\hline
\end{tabular}

f

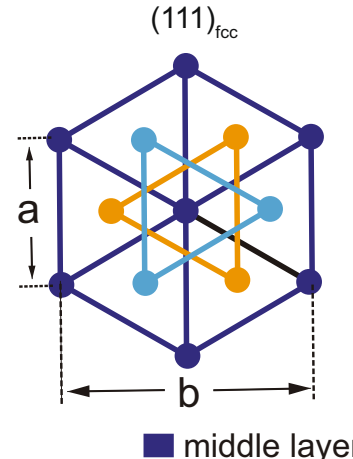

Nucleation

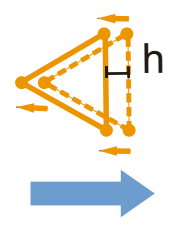

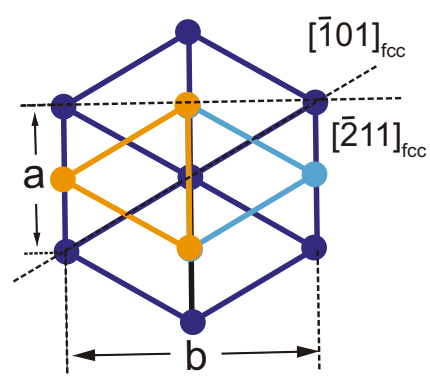

lower layer
Growth
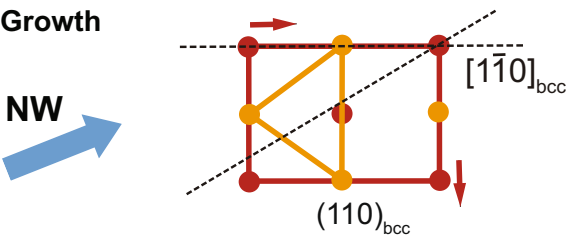

$(110)_{b c c}$
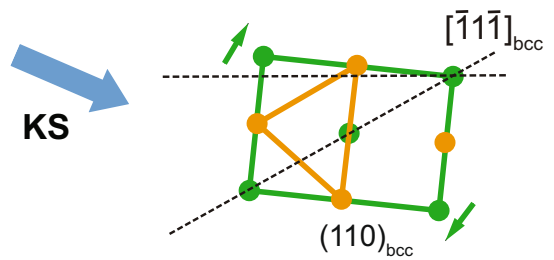

Fig. 1 Experiment details and softness-dependent pathways. a The side view of our experimental setup. The sample cell contains charged PMMA colloidal particles suspended in a density- and refractive index-matched solvent, covered by a semi-permeable membrane with a pore size of $0.22 \mu \mathrm{m}$. lonic AOT micelles with the size of $\sim \mathrm{nm}$ can pass through the membrane, whereas PMMA particles $(\sigma \sim 1.8 \mu \mathrm{m})$ cannot. We first form fcc crystals at a high $C_{\mathrm{AOT}}$ (fcc-stable), then gently change the AOT solution in the reservoir by replacing the one with low $C_{\mathrm{AOT}}$ (bcc-stable). Quick ion exchange (about $10 \mathrm{~s}$ ) triggers an fcc-to-bcc transition inside the sample cell under confocal microscopy observation. $\mathbf{b}$, Equilibrium phase diagram as a function of the particle volume fraction $\phi$ and the AOT concentration $C_{\mathrm{AOT}}$. We perform experiments at $\phi \sim 22 \%$. We illustrate two typical cases of the fcc-to-bcc martensitic transitions from $60 \mathrm{mM}$ AOT to $10 \mathrm{mM}$ (see the orange arrow, weak-driving force) and from 70 to $8 \mathrm{mM}$ (see the red arrow, stronger driving force). c The $C_{\mathrm{AOT}}$-dependence of the shear modulus component $C_{44}$ for parent fcc lattice $\left(C_{44}^{\mathrm{fcc}}\right)$ and final bcc lattice $\left(C_{44}^{\mathrm{bcc}}\right)$. Note the very low value of $C_{44}^{\mathrm{fcc}}$ at $C_{\mathrm{AOT}}=$ $8 \mathrm{mM}$. The blue verticle dotted line indices the fcc-bcc phase line. $\mathbf{d}$ The bcc solid fraction $f_{\mathrm{bcc}}$ as a function of time $t$ after the solution exchange. $t=0$ represents the time when we start to observe $\left(\sim 60 \mathrm{~s}\right.$ after changing $\left.C_{\mathrm{AOT}}\right)$. For the low-modulus, strong-driving-force transition $\left(C_{\mathrm{AOT}} \sim 8 \mathrm{mM}\right)$, the bcc solids fraction increases up to more than $90 \%$ of the system, whereas for the weak-driving-force transition $\left(C_{\mathrm{AOT}} \sim 10 \mathrm{mM}\right)$, it saturates around $50 \%$. e The summary of the kinetic pathways of the fcc-to-bcc transitions observed in our system. The pathways can be classified into two classes (athermal and thermally activated). In a high driving force system, the GB-assisted pathway has a larger contribution to bcc crystallites (70\%) than the in-grain homogeneous pathway (30\%), which are estimated by investigating the formation of bcc crystallites in randomly selected regions of a sample when the amount of bcc solids reaches about $40-50 \%$ of the system. This timing is in the growth stage after the nucleation stage, but bcc solids formed by the ingrain nucleation and GB-assisted nucleation can still be distinguished since it is before they merge. $\mathbf{f}$ The two-step creation process of the NW/KS relationship. First, the upper and lower layers of fcc (111) plane slip relative to the middle layer at the initial nucleation stage (see the inter-layer sliding $h$ ). Then, during further development, two types of intra-layer deformation (see the change of $b / a$ ) lead to the NW and KS relationships.

In our system with a particle volume fraction of $\phi \sim 22 \%$, the fcc-bcc phase-transition line locates near $C_{\mathrm{AOT}} \sim 15 \mathrm{mM}$, and the bcc melting line locates near $C_{\mathrm{AOT}} \sim 6 \mathrm{mM}$, as shown by the phase diagram in Fig. 1b. A rapid decrease of $C_{\mathrm{AOT}}$ from a high concentration (fcc-stable, i.e. $C_{\mathrm{AOT}}=70 \mathrm{mM}$ ) to a low concentration (bcc-stable, i.e. $C_{\mathrm{AOT}}=10 \mathrm{mM}$ ) provides the system with a driving force $\Delta \mu$ of the fcc-to-bcc transition. At the same time, reducing $C_{\mathrm{AOT}}$ (below $15 \mathrm{mM}$ ) softens the parent fcc lattice, as shown by the 
a

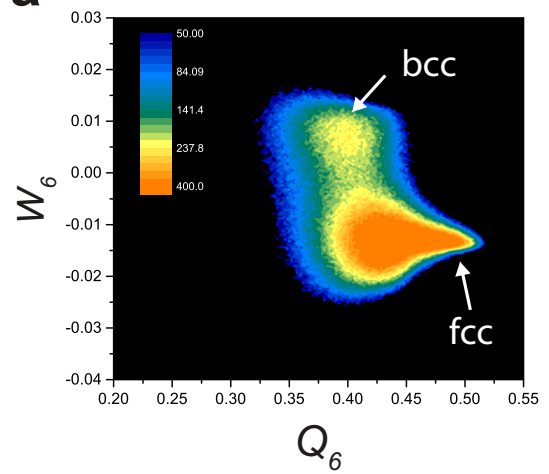

b

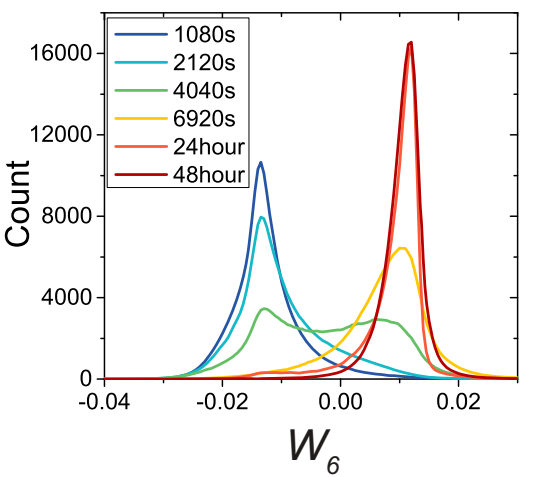

C

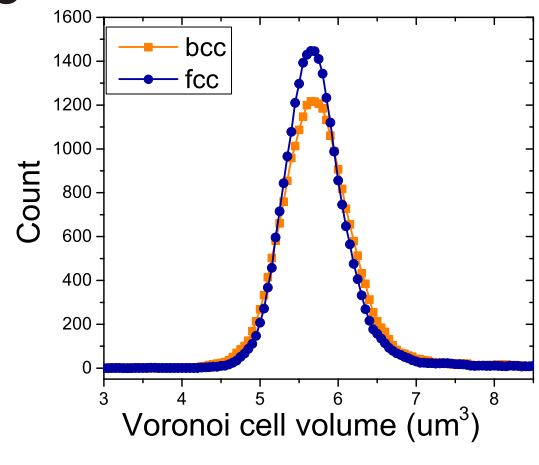

Fig. 2 Illustration of the fcc-to-bcc transition in the 2D order-parameter space. a 2D distribution of particles' local order in the $Q_{6}-W_{6}$ parameter space for the strong-driving force (the data accumulated from $t=0 \mathrm{~s}$ to $t=8000 \mathrm{~s}$ ). From the fcc-solid region of high $Q_{6}$ with negative $W_{6}$, the particles undergo the decrease in $Q_{6}$ and the increase in $W_{6}$ on the $2 \mathrm{D}$ parameter space and eventually transform to bcc solid with a jump of $W_{6}$. We can see that liquid particles $\left(Q_{6}<0.35\right)$ rarely participate in the transformation process. $\mathbf{b}$ The $W_{6}$ distribution of particles at different time. It suggests the effectiveness of $W_{6}$ to distinguish fcc from bcc. c The Voronoi cell volume, which is inversely proportional to the local density, for fcc particles (the parent phase) and bcc particles (the product phase). We can see a tiny density difference $(0.68 \%)$ between the two phases.

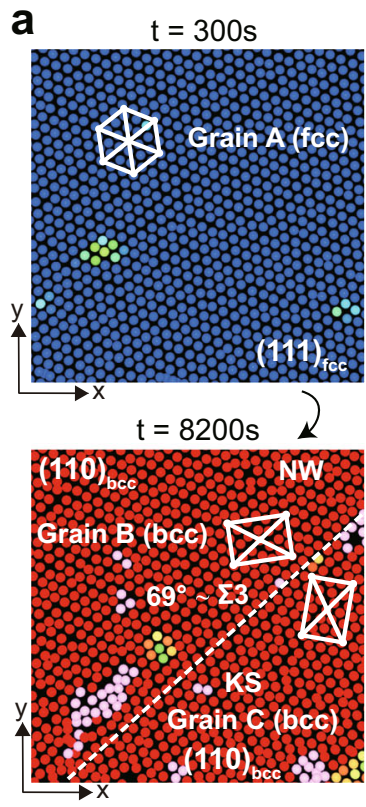

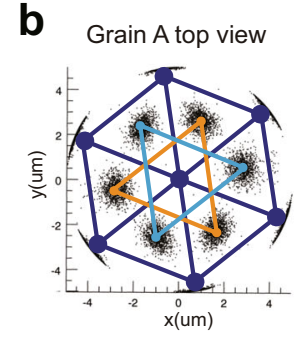

c

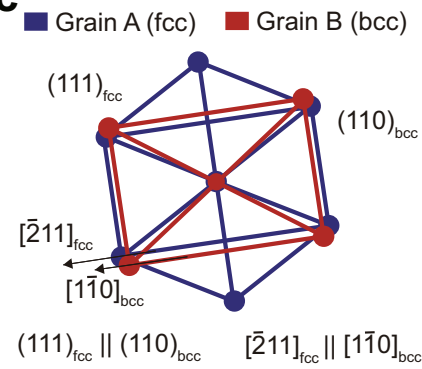

NW relation
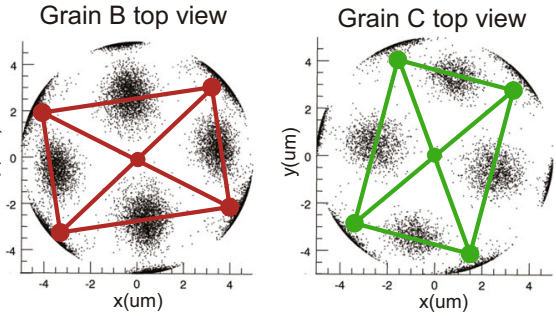

d
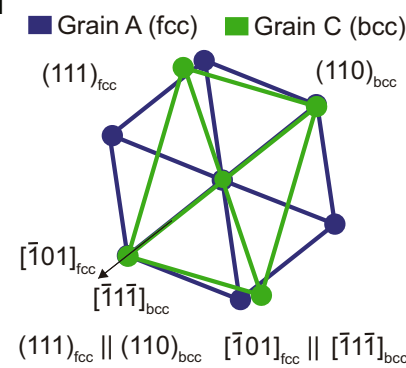

KS relation

Fig. 3 Examples of bcc twinning grains in the product bcc solid. a Creation of the bcc twinning grains $B$ and $C$ from fcc grain A (the inside-grain nucleation). Colour represents the value of $W_{6}$. Pink particles are defect clusters with $Q_{6}<0.35$. $\mathbf{b}$ Representation of the orientational relationship by the bond orientational diagram of each grain. $\mathbf{c}$ The NW relationship between fcc grain A and bcc grain B. $\mathbf{d}$ The KS relationship between fcc grain A and bcc grain $C$. The twinning boundary between grains $B$ and $C$ forms with a tiny angle rotation of the grains.

$C_{\mathrm{AOT}}$-dependence of the shear modulus component $C_{44}^{\mathrm{fcc}}$ and $C_{44}^{\mathrm{bcc}}$ in Fig. 1c (see 'Methods' for $C_{44}$ definition and measurements). We can use this property to probe how the lattice softness affects the microscopic kinetics. At each $C_{\mathrm{AOT}}$, we estimate the effective temperature $T$ in units of the melting temperature $T_{m}$ of the bcc lattice, using the Lindemann criteria: $T \sim \delta L^{2}\left(C_{\mathrm{AOT}}\right) T_{\mathrm{m}} / \delta L^{2}\left(C_{\mathrm{m}}\right)$, where $C_{\mathrm{m}}$ is the AOT concentration close to the bcc melting line at $\phi \sim 22 \%$.

Typically, a rapid jump from $C_{\mathrm{AOT}}=60 \mathrm{mM}$, where fcc is stable, but a small amount of pre-existing bcc exist, to $C_{\mathrm{AOT}}=10$ $\mathrm{mM}$ (indicated by the orange arrow in Fig. 1b), where bcc is stable, produces a weak-driving force of the transition at $T \sim$ $0.65 T_{\mathrm{m}}(10 \mathrm{mM})$. On the other hand, a rapid jump from $C_{\mathrm{AOT}}=$ $70 \mathrm{mM}$ (fcc-stable, pre-existing bcc is less frequent than at
$C_{\mathrm{AOT}}=60 \mathrm{mM}$ ) to $C_{\mathrm{AOT}}=8 \mathrm{mM}$ (see the red arrow in Fig. $1 \mathrm{~b}$ ) produces a stronger driving force (larger $\Delta \mu$ ) but leads to a lower $C_{44}^{\mathrm{fcc}}$. A strong-driving-force and low-modulus thus characterise the resulting transition at $T \sim 0.9 T_{\mathrm{m}}(8 \mathrm{mM})$. Careful observations reveal a variety of dynamics of the fcc-to-bcc transition, depending upon $C_{44}^{\mathrm{fcc}}$ and the degree and type of defects in the initial parent fcc crystal (i.e. defect-free, grain boundaries, and boundary walls).

First, we can notice that the fraction of the produced bcc solids, $f_{\mathrm{bcc}}$ depends on $C_{44}^{\mathrm{fcc}} / \Delta \mu$, as shown in Fig. 1d. The shape of the temporal change of $f_{\mathrm{bcc}}$ is also different between the two cases. The low-modulus, the strong-driving-force condition makes the growth of $f_{\mathrm{bcc}}$ more abrupt. On the other hand, the time taken to reach the final saturation of the bcc solids fraction is similar 
a $\quad t=300 \mathrm{~s}, \mathrm{fcc}$

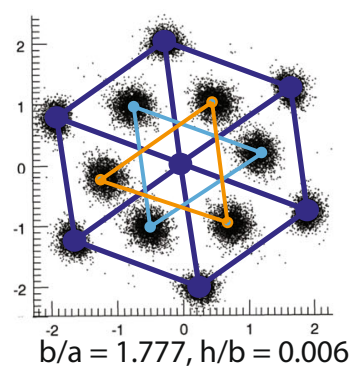

b

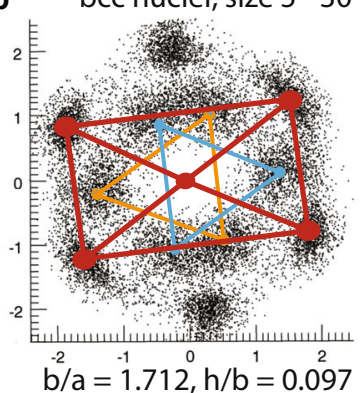

C bcc nuclei, size 30 - 60

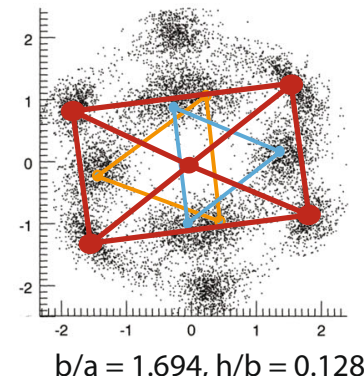

$\mathrm{b} / \mathrm{a}=1.694, \mathrm{~h} / \mathrm{b}=0.128$

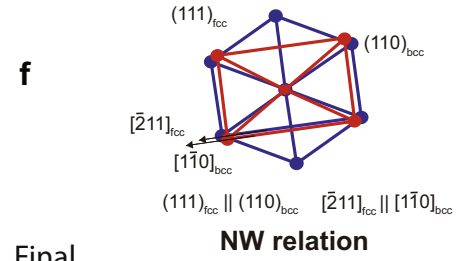

d bcc nuclei, size 60 - 120

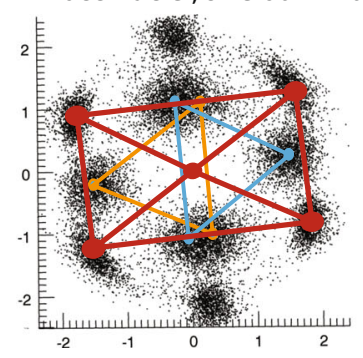

$\mathrm{b} / \mathrm{a}=1.683, \mathrm{~h} / \mathrm{b}=0.144$

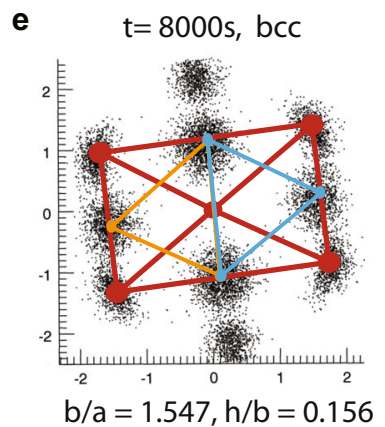

Final

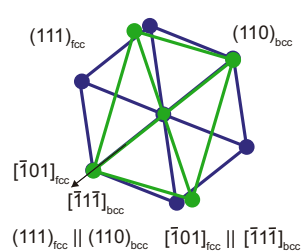

KS relation

Fig. 4 The creation kinetics of NW/KS relationship. Scatter plots are 2D projections of Bond Orientation Order Diagrams (BOODs) of local nuclei. Spheres represent centres of clusters on BOODs, viewed as the averaged lattice structures. Two geometry metrics $b / a$ and $h / b$ are calculated from these averaged lattices, illustrating the transition from fcc $(b / a=1.73, h / b=0)$ to $b c c(b / a=1.41, h / b=0.167)$. a Averaged fcc lattice at $300 s$ after AOT substitution, calculated from a single big fcc grain. The fcc grain at this time is close to the perfect fcc lattice. b-d We select bcc nuclei with different sizes that are generated from the fcc grain in panel a and calculate their averaged lattices. As the size of bcc nuclei increases, the lattice structures change gradually from fcc to bcc. Significant changes will first be seen on $h / b$, caused by inter-layer adjusting. e At $8000 \mathrm{~s}$ after the AOT substitution, the fcc grain we investigate has mostly evolved into bcc solid while keeping the specific orientation relation. The bcc grain at this time will undergo further intra-layer adjustments to modify $b / a$. $\mathbf{f}$ The transition kinetics results in two specific orientation relations between the fcc mother lattice and bcc product lattice: Nishiyama-Wasserman (NW) and Kurdjumov-Sachs (KS) relations.

between the two cases $(\sim 23 \mathrm{~h})$. It implies that the final bcc solids fraction is determined by the number of nucleation sites already existing in the initial parent phase $\mathrm{e}^{1,7}$.

We also find that a system selects the optimal nucleation pathway, depending on the conditions, as summarised in Fig. 1e. For weak-driving-force cases, the athermal nucleation behaviours are dominant. They include the three pathways observed at $10 \mathrm{mM}$, which are initiated from pre-existing bcc nuclei at different sites in the system (inside the fcc grains, on the grain boundaries, and at the flat wall surface). In these cases, the bcc nuclei are already pre-existing in the parent fcc lattice at $C_{\mathrm{AOT}}=$ $60 \mathrm{mM}$, specifically at the triple junction of grain boundaries and at the flat walls surfaces, and their growth is mainly governed by the balance between $A \gamma$, and $\Delta \mu$. These behaviours are much consistent with the athermal features observed in hard materials, such as metals and alloys. On the other hand, surprisingly, we discover that the nucleation behaviour changes from athermal to thermally activated when the parent fcc lattice becomes soft enough (see the two pathways at $8 \mathrm{mM}$ ): spontaneous nucleation of new bcc crystallites are observed inside the fcc parent lattice and near the premelting grain boundaries. For these two pathways, we find that the flow in the sample during the nucleation (measured by a particle tracking of $20 \mathrm{~s}$ that is about ten times longer than the particles' Brownian time) is negligible $(<2 \mathrm{~nm} / \mathrm{s})$, indicating that the pathways belong to thermally activated nucleation in the absence of applied stresses.
We note that all the nucleation pathways in Fig. 1e are diffusionless processes, and the produced bcc crystals have the same Nishiyama-Wassermann (NW) ((111) fcc $\|(110)_{\mathrm{bcc}}$, $\left.[\overline{2} 11]_{\mathrm{fcc}} \|[1 \overline{1} 0]_{\mathrm{bcc}}\right)$ or Kurdjumov-Sachs (KS) $\left((111)_{\mathrm{fcc}} \|(110)_{\mathrm{bcc}}\right.$, $\left.[\overline{1} 01]_{\mathrm{fcc}} \|[\overline{1} 1 \overline{1}]_{\mathrm{bcc}}\right)$ orientation relations with the parent fcc solid. We note that these two famous orientational relationships characterising the matching between the directions and planes of the parent fcc phase and those of the product bcc phase were established for martensitic transitions of steels (see, e.g. ref. ${ }^{4}$ ). Moreover, we find twinnings of the product bcc grains inside one large parent fcc grain as commonly observed in hard materials (Fig. 3), which has NW and KS orientation relations with the parent fcc grain. Interestingly, we observe two sequential elementary steps to establish the orientational relation: first, the upper and lower layers of the fcc (111) plane slip relative to the middle layer (see the inter-layer sliding $h$ from the left to middle panel of Fig. 1f), which mainly happens at the initial nucleation stage (see the evolution with respect to the nuclei size in Fig. 4). It is then followed by the two types of intra-layer deformation of the hexagonal plane at the growth stage, leading to the NW and KS relationships (see the two types of mode that change $b / a$ in the most right panels of Fig. 1f). This is the first experimental illustration for the transformation kinetics at a microscopic level. Note that both the parent fcc (111) and final bcc (110) planes are parallel to our samples' boundary wall. However, the orientation relation is established inside the sample already when the nucleus 

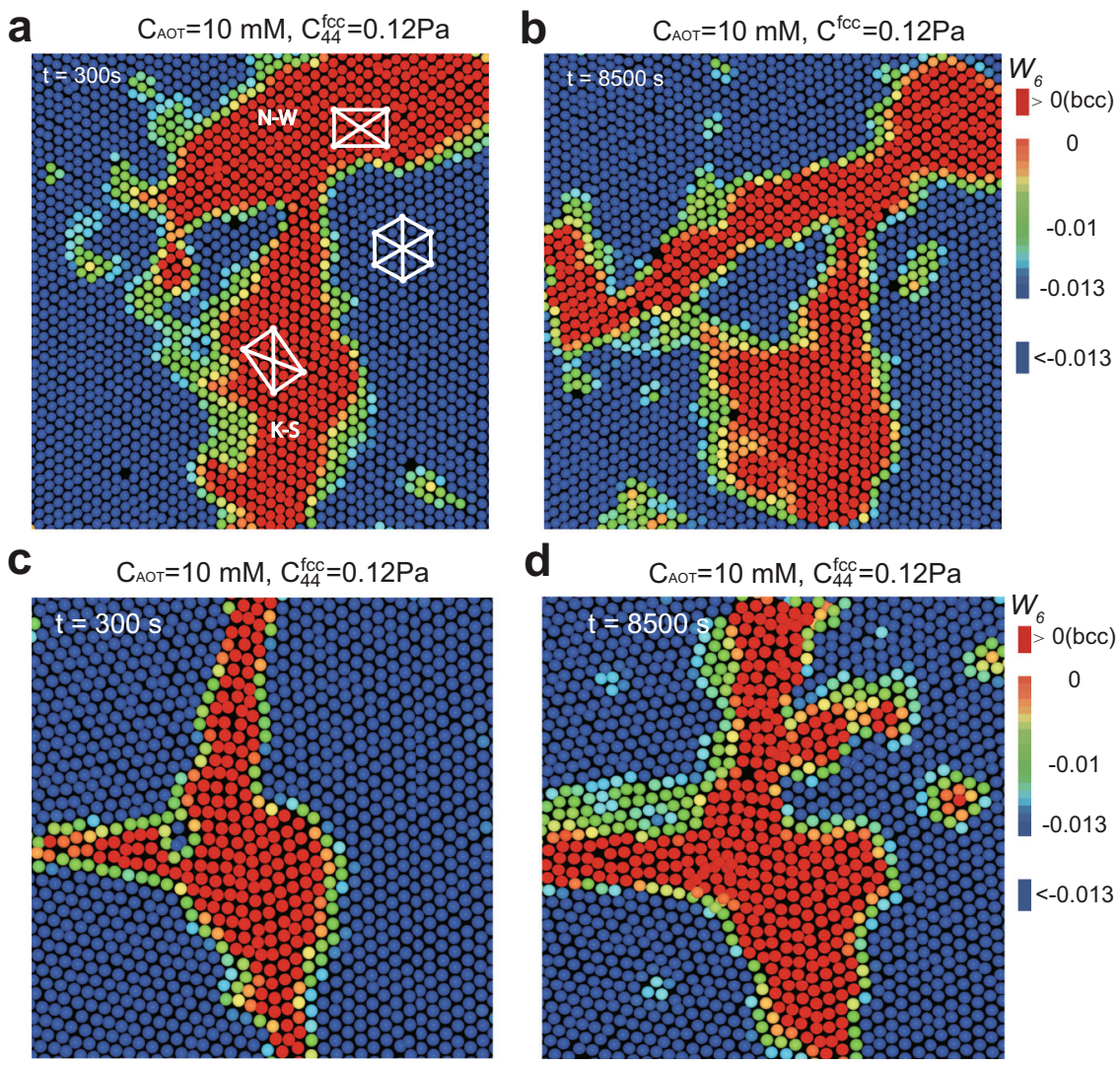

Fig. 5 Pre-existing bcc nuclei inside the fcc grain and at the triple junction of grain boundaries. a Pre-existing bcc nuclei at the parent fcc grain at $C_{\text {aot }} \sim 60 \mathrm{mM}$. They already have NW or KS orientation relation with the fcc lattice. $\mathbf{b}$ The little development of the bcc nuclei under the weak-driving force $\left(C_{\text {aot }} \sim 10 \mathrm{mM}\right.$ ) because of the absence of surface-energy and strain-energy reductions. $\mathbf{c}$ Pre-existing bcc nuclei at the triple junction of grain boundaries at $C_{\text {aot }} \sim 60 \mathrm{mM}$. d The little development of the bcc nuclei under the weak-driving force $\left(C_{\text {aot }} \sim 10 \mathrm{mM}\right)$, because of the absence of significant surface-energy and strain-energy reductions.

is small, suggesting that the specific orientation relation is a consequence of an intrinsic trend to reduce the diffusionless transformation's energy cost, even for ultra-soft materials. We also note that the interface between the parent fcc phase and the product bcc phase in our soft colloidal systems is not as sharp as in hard materials (we will discuss this point in detail later).

For the two classes (athermal and thermally activated) of nucleation behaviours listed in Fig. 1e, we can further divide them into (i) inside-grain nucleation, (ii) grain boundary-assisted nucleation, and (iii) wall-assisted nucleation. In the following, we discuss them one by one, focusing on the parent lattice's softness and how the system chooses the most appropriate pathway to overcome the nucleation barrier efficiently.

Inside-grain nucleation. First, we discuss the inside-grain nucleation (type (i)). For the low-driving-force case, classical models suggest the heterogeneous nucleation pathway characterised by nuclei pre-existing already above the transition temperature, stabilised by defects such as dislocations and stacking faults. This athermal pathway is selected when $E_{\text {strain }}$ is much larger than $k_{\mathrm{B}} T$ and dominant ${ }^{1,7}$. As expected, we find that there are pre-existing bcc nuclei at $C_{\mathrm{AOT}} \sim 60 \mathrm{mM}$ (above the bccfcc phase line, $C_{\mathrm{AOT}} \sim 15 \mathrm{mM}$ ), and its growth is limited under the weak-driving-force $\left(C_{\mathrm{AOT}} \sim 10 \mathrm{mM}\right.$, see Fig. $\left.5 \mathrm{a}, \mathrm{b}\right)$ because of the absence of surface- and strain-energy reduction in Eq. (1). Interestingly, thermal fluctuations cause the slow migration of the pre-existing bcc nuclei, as shown in Fig. 5b, which can be thought of as an interface roughening effect. We note that the flow induced in our samples by the AOT substitution (measured by a particle tracking of $20 \mathrm{~s}$ that is about ten times longer than the particles' Brownian time) is negligible $(<2 \mathrm{~nm} / \mathrm{s})$. Moreover, we do not find nucleation sites inside the parent fcc grain other than the pre-existing ones, indicating the absence of stress-driven nucleation induced by a sample flow. Together with the stressdriven nucleation in the presence of a sample flow reported in ref. ${ }^{14}$, our observation highlights the significant role of stress on the martensitic transition under a low-driving force.

Surprisingly, bcc nuclei can be formed through thermal activation when the parent $\mathrm{fcc}$ lattice is soft enough. We can prepare defect-free grains without pre-existing bcc nucleus inside at $C_{\mathrm{AOT}} \sim 70 \mathrm{mM}$, which is above the fcc-bcc phase line and higher than $60 \mathrm{mM}$. At $C_{\mathrm{AOT}} \sim 8 \mathrm{mM}$ (see the red arrow in Fig. 1b), we observe the thermally activated homogeneous nucleation kinetics inside dislocation-free grains, as illustrated in Figs. 6a, b and Supplementary Movies S1 and S2. Supplementary Movie S1 has a larger dimension $(150 \mu \mathrm{m} \times 150 \mu \mathrm{m} \times 100$ $\mu \mathrm{m}$ ) but a lower time resolution (the time interval of $\sim 300 \mathrm{~s}$ ), whereas Supplementary Movie S2 has a lower dimension (150 $\mu \mathrm{m} \times 150 \mu \mathrm{m} \times 20 \mu \mathrm{m}$ ) but a higher time resolution (the time interval of $\sim 2.5 \mathrm{~s}$ ). Large orange spheres in the movies represent bcc particles, whereas large blue spheres represent defects or their clusters $\left(Q_{6}<0.35\right)$. Note that particles with $Q_{6}<0.35$ in soft colloidal systems are mostly highly distorted (defective) solid structures that contain a small amount of liquid-like $\left(Q_{6}<0.25\right)$ particles $^{41}$

Initially (at $C_{\mathrm{AOT}} \sim 70 \mathrm{mM}$ ), the parent fcc lattice is defect-free and stable. It is characterised by the negative value of $W_{6}\left(W_{6} \sim\right.$ -0.013 ) reflecting the fcc structure and also by its homogeneity 
a
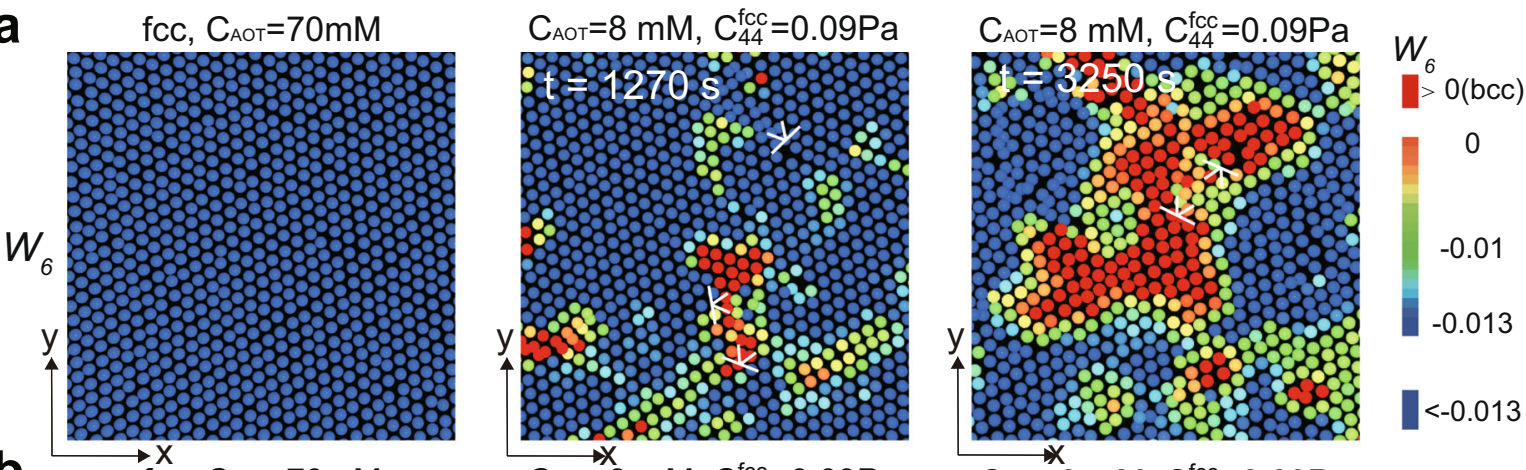

b

$\mathrm{fCC}, \mathrm{C}_{\mathrm{AOT}}=70 \mathrm{mM}$

$\delta L$
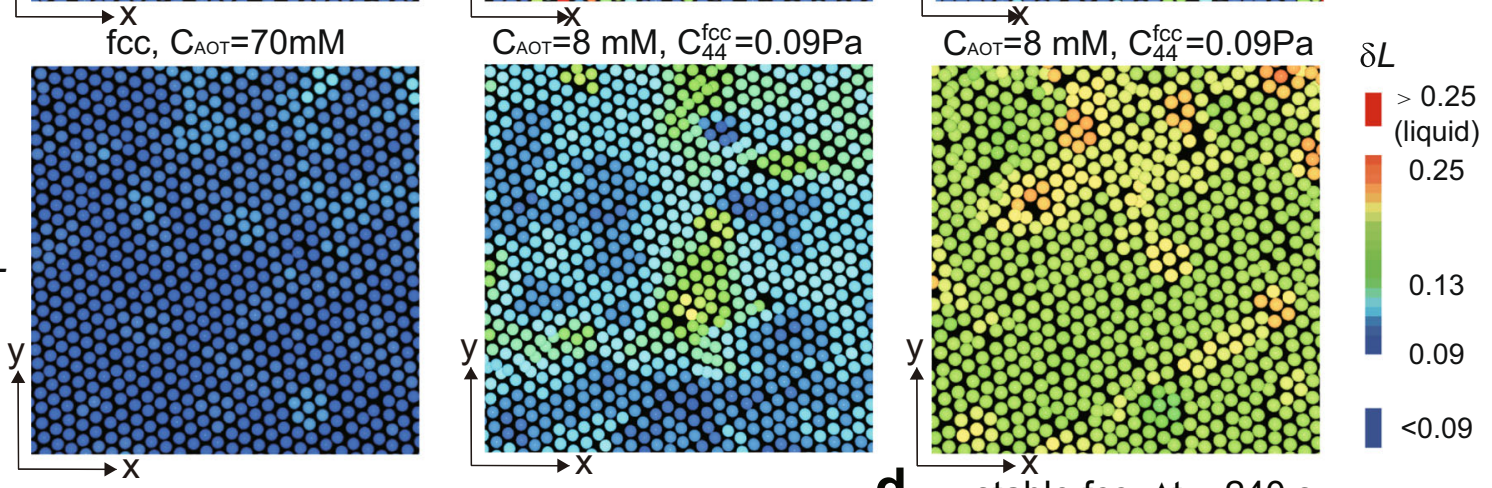

C

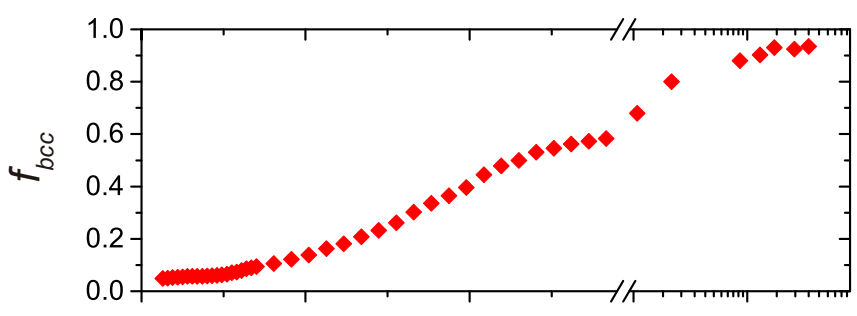

d
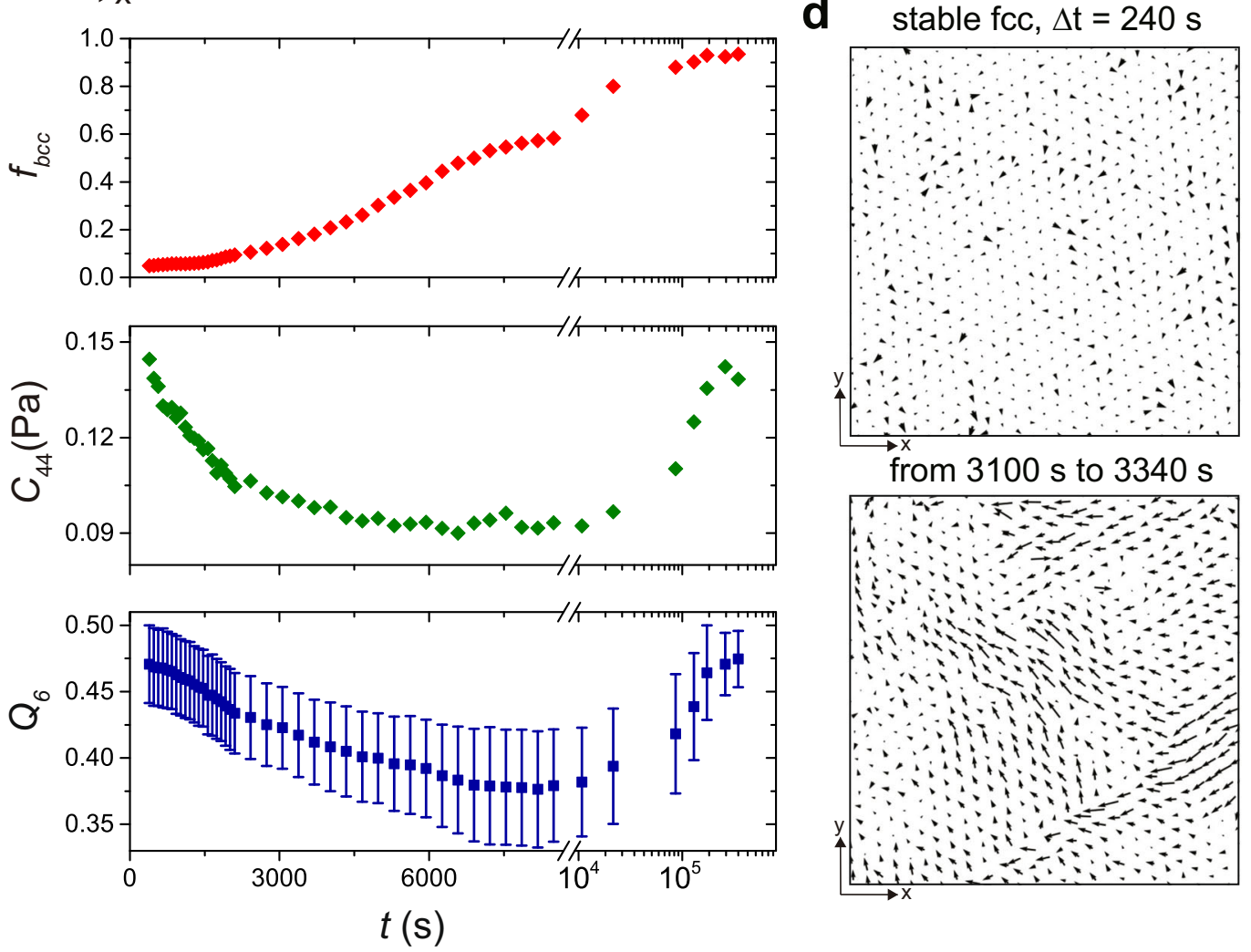

Fig. 6 The thermally activated inside-grain homogeneous nucleation for low-modulus, strong-driving-force transition. a Colour plot of $W_{6}$ of particles undergoing the fcc-to-bcc transition (red particles represent bcc; dislocations are specified). The bcc critical nucleus $\left(t \sim 3250 \mathrm{~s}, W_{6} \geq 0\right)$ appear in the region containing defects $(t \sim 1270 \mathrm{~s}$ ), which spontaneously forms in the defect-free fcc lattice. $\mathbf{b}$ The colour plot of the Lindemann parameter $\delta L$ of particles undergoing the fcc-to-bcc transition, the regions with large Lindemann parameter $\left(t \sim 1270 \mathrm{~s}\right.$ ) well correspond to the regions of large $W_{6}$ fluctuations (small bcc nuclei) in a. c Time evolution of the fraction of bcc solids, $f_{b c c}$, the component $C_{44}$ of the shear modulus, and the average $Q_{6}$ of the system. The error bar of $Q_{6}$ represents the standard deviation. $\mathrm{C}_{44}$ before the truncation point is calculated for the parent fcc lattice, whereas the one after the truncation point is calculated for the product bcc lattice. $Q_{6}$ is averaged over all particles in the system. In the nucleation stage $(t<3500 \mathrm{~s})$, the formation of defects seen by the lowering of $Q_{6}$ coincides well with the drop of the shear modulus $C_{44}$. In the late growth stage $\left(t>7.5 \times 10^{4} \mathrm{~s}\right)$, the slow eradication of defects seen by the growth of $Q_{6}$ also coincides well with the increase of $C_{44}$. $\mathbf{d}$ The displacement field of particles (at $\Delta t \sim 240 \mathrm{~s}$ ) of the initial fcc lattice (see the top panel, $t<0$ ) and that of particles after the formation of a critical bcc nucleus ( $t \sim 3250 \mathrm{~s}$, bottom panel). Due to the small shear modulus $C_{44}^{\mathrm{fcc}}$, the bcc nuclei are created by displacive heterogeneities corresponding to the slipping motion of particles. The particles shown in panel $d$ are the same as those in (a and $\mathbf{b}$ ). 

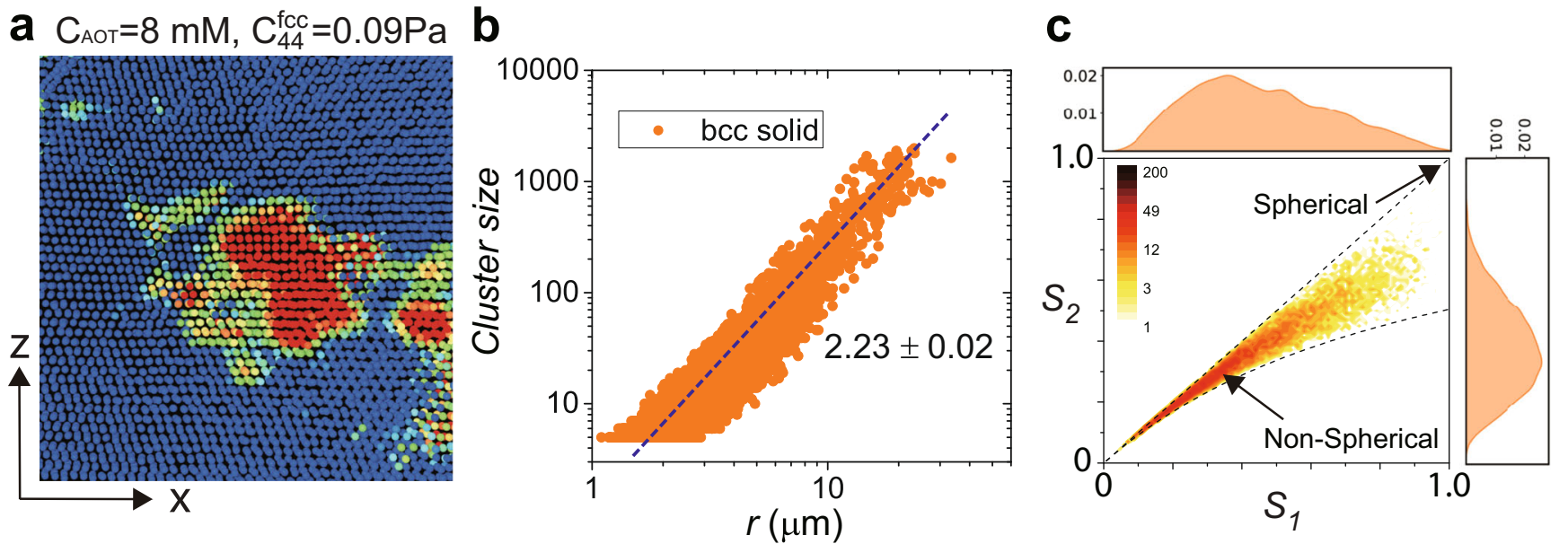

Fig. 7 Anisotropic shape and ramified interface of in-grain nucleated bcc nuclei. a The $x-z$ slice of in-grain nucleated bcc nuclei, where we can see the anisotropic shape with the ramified interface, similar to the one in the $x-y$ slice (Fig. 6a). b The distribution of the mass-gyration radius in 3D for in-grain nucleated bcc nuclei. The fractal dimension of $d \sim 2.23$ manifests the ramified feature of the interface. $\mathbf{c}$ Density plot of bcc nuclei clusters on the shapefactor space $\left(S_{1}, S_{2}\right)$, directly indicating their non-spherical shape. The point $S_{1}=S_{2}=1$ represents a perfectly spherical shape. We can see that most bcc clusters belong to the non-spherical region. The most probable position is around $(0.35,0.3)$.

due to the absence of defects (see the most left panel of Fig. 6a). The parent phase also has a small value of the Lindemann parameter, $\delta L$, of each particle, as shown in the most left panel of Fig. 6b. Then, after the strong-driving force towards the bcc phase is imposed by a jump of $C_{\mathrm{AOT}}$ to $\sim 8 \mathrm{mM}$, defects spontaneously develop as fluctuations of the fcc lattice, which softens the nearby lattice sites and leads to the formation of small bcc nuclei at $t=$ $1270 \mathrm{~s}$ (see the red particles in the middle panel of Fig. 6a). Correspondingly, the Lindemann parameter also increases around the defects (see the green region with larger $\delta L$ in the middle panel of Fig. 6b). The spontaneous fluctuations keep growing and eventually lead to the formation of the bcc phase's large nucleus (see the most right panels of Fig. $6 \mathrm{a}, \mathrm{b}, t=3250 \mathrm{~s}$ ).

We show the temporal changes in the bcc solid fraction $f_{\mathrm{bcc}}$ the shear modulus component $C_{44}$, and the structure order $Q_{6}$, in the three panels in Fig. 6c, respectively, from top to bottom. They provide the following physical picture of the inside-grain homogeneous nucleation: The lowering of $C_{44}$ and $Q_{6}$ in the nucleation stage $(t<3500 \mathrm{~s})$ generates heterogeneities in the displacement field in the parent lattice, triggering the nucleation of the product phase. This physical picture is strongly supported by the striking difference in the displacement field between the stable fcc lattice (the top panel of Fig. 6d) and the fcc crystal coexisting with the nucleated bcc crystal (the bottom panel of Fig. 6d).

Surprisingly, we observe the increase of $C_{44}$ and $Q_{6}$ in the very late stage of bcc growth (Fig. $6 \mathrm{c}$, at $20 \mathrm{~h}$ later), which reflects the slow eradication of the lattice defects that are created during the structural transformation. This behaviour again illustrates the critical role of lattice defects in the fcc-to-bcc transformation. Facilitated by thermally excited lattice defects, the in-grain bcc nuclei have a ramified interface (the most right panel of Fig. 6a) with anisotropy (Fig. 7), which should be a genuine feature of low-barrier solid-tosolid transition in the absence of applied stresses. This ramified interface produced by thermally activated nucleation distinctly contrasts with the sharp, twinning interface typically observed in metals.

The above finding reveals that nucleation pathways can be softness-dependent. It demonstrates the critical role of the thermalexcitation of defects for ultra-soft lattice in the nucleation process, which has not been reported before. This mechanism should be a new family member of solid-to-solid transitions characteristic to soft systems. Concerning this, it is worth mentioning that previous works in colloidal systems reported the liquid state-mediated nucleation kinetics, in which the nucleation involves the diffusive motion of particles without specific orientational relation to the parent phase ${ }^{14,18,31}$. The discovery of diffusionless defect-mediated spontaneous nucleation in addition to the diffusive one deepens our understanding of solid-solid transformations in soft, defect-free crystals.

Grain boundary-assisted nucleation. It has been widely believed that grain boundaries (GBs) play pivotal roles in determining crystalline materials' physical properties and kinetic behaviours ${ }^{42}-44$. For example, the triple junction of GBs has been widely recognised to be one of the most preferred nucleation sites in martensitic transitions of metals. As expected, we indeed find similar pre-existing bcc nuclei at the triple junction $\left(C_{\mathrm{AOT}} \sim 60 \mathrm{mM}\right.$, see Fig. 5c, d). However, how GB assists the nucleation, e.g. whether through its roughening or via premelting caused by thermal activation, is poorly understood.

Here, we show that the premelting of GBs greatly facilitates the grain boundary nucleation because it leads to a small bcc-liquid interface tension. As shown by the appearance of thick defective layers $\left(Q_{6}<0.35\right.$, pink colour $)$ in Fig. 8a, the GB premelts under thermal fluctuations when the parent fcc lattice is soft enough $\left(C_{\mathrm{AOT}} \sim 8 \mathrm{mM}, t=300 \mathrm{~s}\right)$. Because the bcc-liquid interface tension, $\gamma_{\text {bcc-liquid, }}$ is smaller than the bcc-fcc one, $\gamma_{\text {bcc-fcc }}$ (characterised by the contact angle of $\sim 35^{\circ}$ in left panel of Fig. 8b.), the premelting of GB helps to reduce the interface energy term $A \gamma$ in Eq. (1). The premelted layers can also facilitate the solid's displacive movement, which can reduce the strain energy. Thus, we observe heterogeneous nucleation of bcc solid in the melt and its further growth (see the panels at $t \sim 1740 \mathrm{~s}$ and $t$ $\sim 3060 \mathrm{~s}$ of Fig. 8b).

At $C_{\mathrm{AOT}} \sim 10 \mathrm{mM}$, on the other hand, GB does not premelt and only shows a roughening behaviour, as shown in Fig. $8 \mathrm{c}(t=$ $300 \mathrm{~s}$ ). Thus, even after a much longer time (see the panels at $t \sim$ $1740 \mathrm{~s}$ and $t \sim 7220 \mathrm{~s}$ of Fig. 8d), the tiny bcc nuclei still stuck around the GB because the roughening behaviour causes a little surface-energy reduction in Eq. (1).

Under the strong-driving force at $C_{\mathrm{AOT}} \sim 8 \mathrm{mM}$, the mechanism of GB-assisted nucleation and growth accounts for about $70 \%$ 


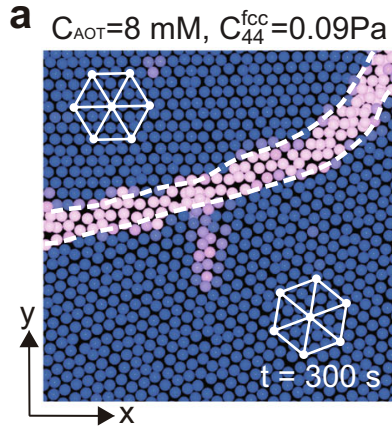

C $_{\mathrm{C}_{\mathrm{AOT}}=10 \mathrm{mM}, \mathrm{C}_{44}^{\mathrm{fcC}}=0.12 \mathrm{~Pa}}$

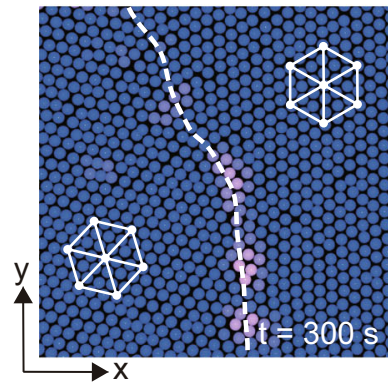

b

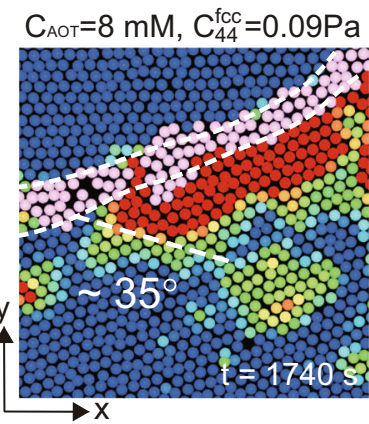

d

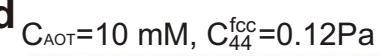

0.45

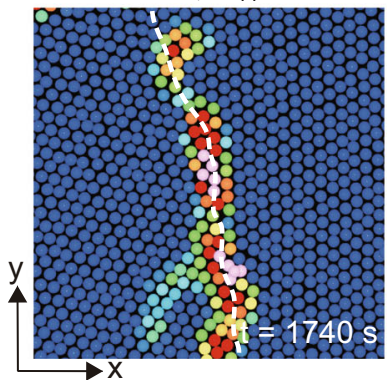

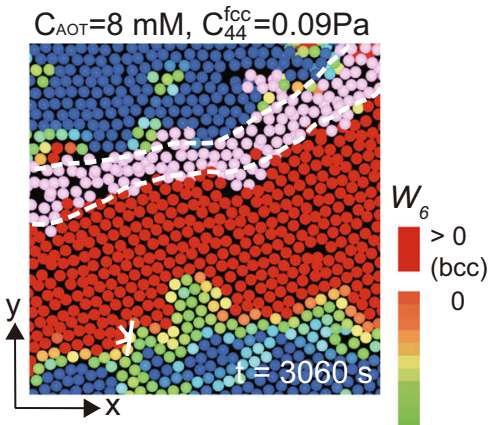

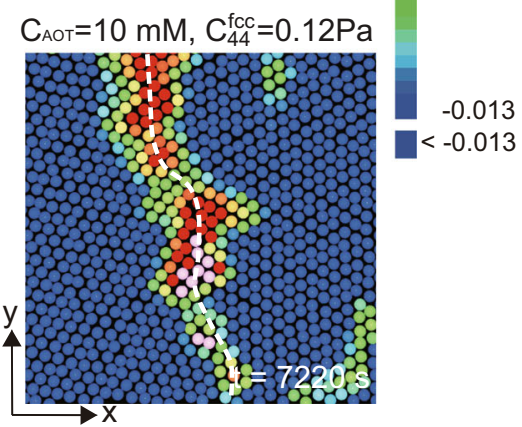

Fig. 8 The grain boundary-assisted nucleation accompanied by premelting of the grain boundary. $\mathbf{a}, \mathbf{b}$ Premelting of the grain boundary $\left(C_{\mathrm{AOT}}=8 \mathrm{mM}\right.$, $t \sim 300 \mathrm{~s}$ after the AOT substitution) happens for the low-modulus transition in (a), leading to the heterogeneous nucleation initiating from it $\left(C_{\mathrm{AOT}}=\right.$ $8 \mathrm{mM}, t \sim 1740 \mathrm{~s}$ and $t \sim 3060 \mathrm{~s}$ in (b), respectively). Bcc solid wets the liquid with an equilibrium contact angle of about $35^{\circ}$. The premelting also facilitates the displacive motion of the solid. $\mathbf{c}$, $\mathbf{d}$ The premelting behaviour is absent at $C_{\mathrm{AOT}}=10 \mathrm{mM}$, resulting in a small surface-energy reduction. Therefore, there is little bonus for the bcc nuclei generation and development.

of the bcc solids formation (see 'Methods' for the measurement details). This GB-assisted nucleation pathway we observed in soft colloidal crystals should be relevant to martensitic transitions close to the melting temperature, i.e. near the triple point at which the liquid and the two solid phases coexist, as long as the GB premelting can take place. This mechanism possibly paves the way for applying the long-existing GB engineering to the control of solid-to-solid transitions.

Wall-assisted growth. For the weak-driving-force transition at $C_{\mathrm{AOT}} \sim 10 \mathrm{mM}$, the parent fcc lattice is not soft enough, and most GBs do not premelt; thus, the pre-existing bcc is the primary source of nucleation. In this case, the inside-grain and GBassisted growth modes are not significantly facilitated. However, we find another pathway that can facilitate the growth of preexisting nuclei: the wall-assisted growth, leading to the final bcc fraction of around $50 \%$.

The smooth flat wall has lower interface tension with bcc than $\mathrm{fcc}^{21}$, i.e. $\gamma_{\text {bcc-wall }}<\gamma_{\text {fcc-wall. }}$. We can confirm this by the $\sim 60^{\circ}$ wetting angle of a small bcc nucleus, as shown in Fig. 9a $(t=$ 930 s). The wetting effect reduces the surface-energy term $A \gamma$ in Eq. (1), which promotes the further development of the bcc nucleus (Figs. 9c, d, at $t=6580 \mathrm{~s}$ ).

Now, a natural question arises: if the wall-bcc interface has smaller interface tension and the parent lattice is soft enough, does wall-assisted growth become the dominant mechanism? The answer is no. It is because that the GB-premelting has a more dominant effect than the smooth wall (Fig. 10). Usually, the grain boundary ends at the system's boundary wall, and the grain boundary-assisted growth accompanied by premelting has a lower surface energy than the wall-induced growth, because $\left(\gamma_{\text {bcc-wall }}-\gamma_{\text {fcc-wall }}\right) / \cos \theta_{1} \sim$ $\gamma_{\mathrm{bcc}-\mathrm{fcc}} \sim\left(\gamma_{\mathrm{bcc}-\text { liquid }}-\gamma_{\mathrm{fcc}-\text { liquid }}\right) / \cos \theta_{2}\left(\theta_{1} \sim 60^{\circ}\right.$ in Fig. $9 \mathrm{a}$ and $\theta_{2} \sim 35^{\circ}$ in Fig. 8b). Thus, wall-induced growth rarely happens when
GB-premelting is present. On the other hand, when GB does not premelt, it can effectively be regarded as a rough wall, so that wallassisted growth on a smooth flat wall should be more preferred.

\section{Discussions and outlook}

In addition to the non-thermal nucleation pathways widely observed in hard materials, the martensitic transition kinetics of soft colloidal crystals has revealed the previously unknown nucleation pathways, such as the thermally activated in-grain nucleation, GB-premelting-assisted nucleation, and wall-assisted growth. We discuss the generality of these results for each of these three pathways, focusing on how the energy barrier is reduced, reflecting the system's character.

For the in-grain homogeneous nucleation, the low shear modulus and the absence of liquid intermediates suggest that the parent lattice is likely near the mechanical instability point without exceeding its melting point. In other words, a weak superheating ability, where the superheating limit of the system is located above but very close to the melting point, should be the critical requirement for this type of solid-solid transformation. More specifically, we assume that at least one of the shear modulus components becomes zero at the superheating limit of a solid state, leading to the melting of the solid into a liquid barrierlessly. Thus, this nucleation pathway requires a weak superheating ability as well as a suitably soft lattice modulus before melting. With a weak superheating ability, defects are thermally excited frequently near the melting point, and they serve as the primary source of nucleation sites. We note that the superheating ability is closely related to the potential softness and density of the system, as well known. From these considerations, we expect that the in-grain homogeneous nucleation possibly exists in systems with soft and relatively long-range overlapped potentials (e.g. charge-, 

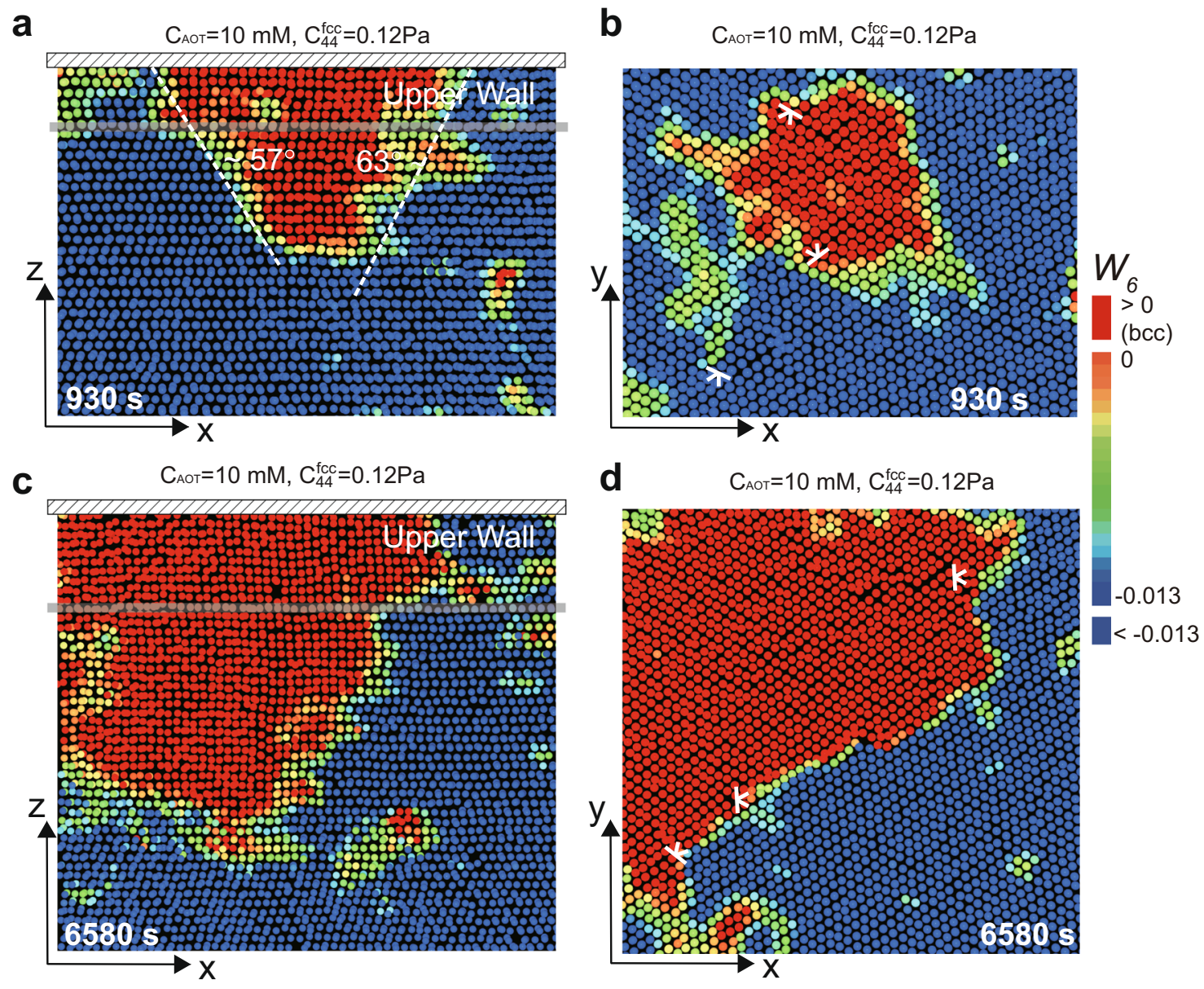

Fig. 9 Wall-assisted growth for pre-existing nuclei observed at the weak-driving force. $\mathbf{a}, \mathbf{b}$ Small pre-existing nuclei formed on the $x-z$ and $x-y$ planes of a wall at $t=930 \mathrm{~s}$. The smooth wall wets the bcc solid with an equilibrium contact angle of about $60^{\circ}$. c, $\mathbf{d}$ The wall-assisted growth corresponding to (a and $\mathbf{b}$ ), respectively, due to the surface-energy reduction of the wall-induced wetting.

dipole- and quadrupole-related interactions), solution-based materials (such as polyelectrolyte and charged proteins), and highly compressed soft polymers.

In the GB-premelting-assisted nucleation, a small surfaceenergy term in the barrier plays a critical role. Because thermalexcitation (rather than applying stress) is still the reason for this nucleation pathway, a lower modulus of the parent lattice and a higher driving force of the solid-to-solid transition reducing the nucleation barrier could also facilitate this process. In this nucleation pathway, the GB-premelting triggers the generation of small nuclei, and their growth is controlled by the balance between the surface energy, strain energy, and chemical-potential differences. Accordingly, a weak superheating ability is unnecessary for this pathway; instead, the GB-premelting alone is primarily responsible. We expect this mechanism to be generally realised because the increase in temperature could trigger the GB premelting before the bulk melting happens. Accordingly, besides soft systems with a weak superheating ability, such a kinetic pathway is likely to be seen in a broad class of materials with a high-temperature triple point (of a liquid and two solid states $)^{45,46}$. We expect that this pathway is operative when the pressure or temperature is close to the triple point, which can happen, e.g. in some organic materials.

The wall-induced growth is similar to the GB-premeltingassisted nucleation in the sense that the reduction of the surfaceenergy term in the barrier plays a crucial role. So, a weak superheating ability is not a necessary condition for this pathway. Moreover, a lower modulus of the parent lattice and a higher driving force of the solid-to-solid transition also help facilitate this pathway. Accordingly, this pathway is also likely to be seen in the systems mentioned above for the GB-premelting-assisted nucleation.

In these three pathways found in our soft colloidal systems, we observe interesting morphologies of the child bcc phase. Firstly, inside one grain of the parent solid, we find nearly $70^{\circ}$ twinning grain boundary of the child bcc phase, which have NW and KS relationships with the parent solid, respectively. Such a morphology during the transition obviously reduces the interface energy between the parent phase and the child phase (two bcc grains that have the same NW or KS relationship with the parent solid could form a grain boundary that is less close to a coherent state, Fig. 3). Next, the interface is not as sharp as in hard materials, which may be regarded as an interface roughening effect due to the low modulus of soft systems. Moreover, the grain boundary networks (large-angle grain boundaries and triple junctions) in the parent phase are retained during the transition, which also reduces the interface energy cost of the transition.

We hope that these kinetic pathways and morphologies found in soft materials deepen the theoretical understanding of the physical mechanism of solid-to-solid transformations and pave the way to control the diffusionless solid-solid transformations, 


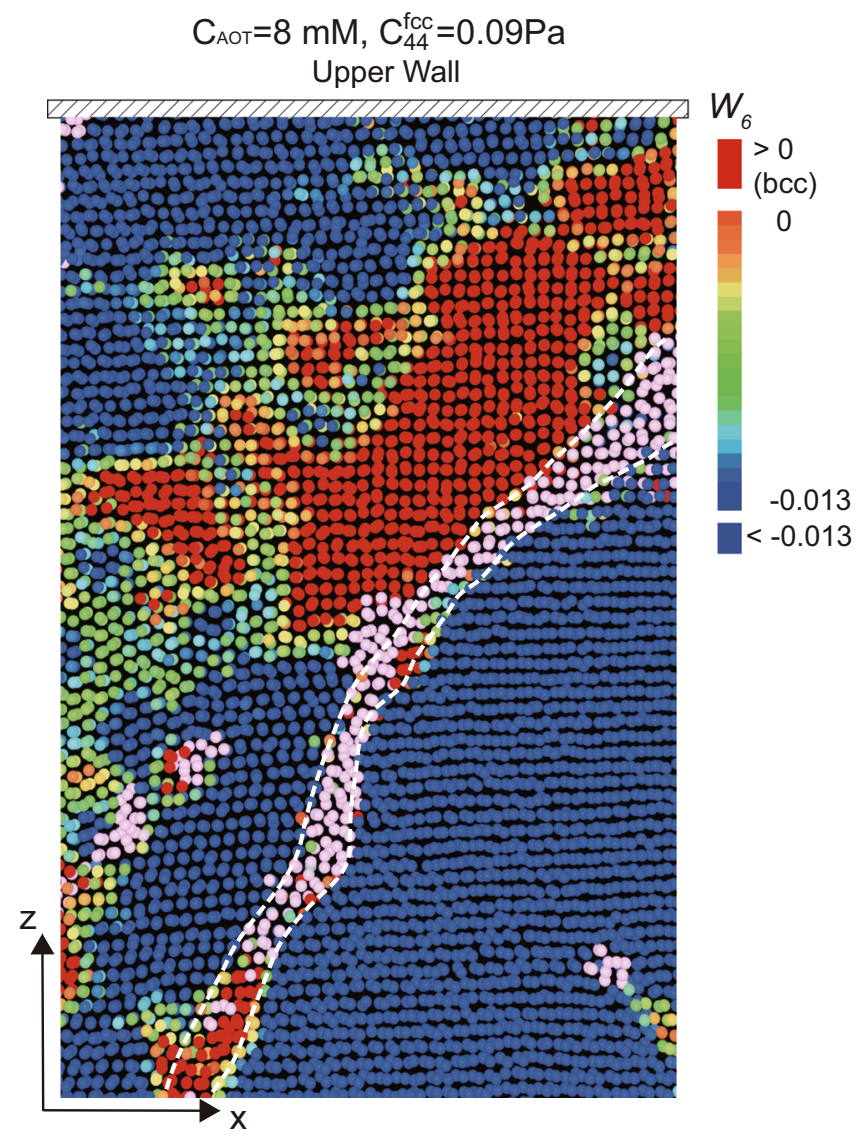

Fig. 10 Rare realisation of wall-assisted growth for the strong-driving

force. The $x-z$ image suggests that wall-assisted growth is not favoured at the strong-driving force. Usually, the grain boundary ends at the wall, and the premelted grain boundary has a more predominant effect than the smooth wall. Accordingly, wall-assisted growth is rarely observed under a strong-driving force.

which should be broadly applicable to crystals of numerous materials.

\section{Methods}

Samples. We suspend $\mathrm{DiIC}_{18}$ dyed and poly hydroxystearic acid polymers grafted poly(methyl methacrylate) (PMMA) colloids (diameter $\sigma=1.8 \mu \mathrm{m}$, polydispersity $\sim 2.5 \%$, https://www2.ph.ed.ac.uk/ abs/) in a mixture of the non-polar solvents (tetrachloroethylene +hexachlorobutadiene + decahydronaphthalene, Aldrich), whose refractive index and density are precisely matched with those of particles. The addition of AOT (sodium di-2-ethylhexyl sulfosuccinate, Aldrich) into the colloidal suspensions produces ionic AOT micelles that can change the interparticle potential ${ }^{36,37}$. The PMMA particles are negatively charged in our system and interact with each other via weakly screened Coulomb repulsion, $u(r)=$ $\alpha \exp [-\kappa \sigma(r / \sigma-1)] /(r / \sigma)$ for $r>\sigma$, with $1 / \kappa$ being the Debye screening length and $\alpha$ being the effective interaction strength in the suspension that can be adjusted by the AOT concentration ${ }^{36,37}$. By reducing the AOT concentration at a constant particle volume fraction $\phi$, we can change the stable solid structures from fcc to bcc, as shown by the phase diagram in Fig. $1 \mathrm{~b}$.

Special sample cell. We design a new sample cell (length $=6 \mathrm{~mm}$, width $=1.5$ $\mathrm{mm}$, depth $=0.1 \mathrm{~mm}$ ) with a semi-permeable membrane (Fig. 1a) ${ }^{47}$. The bottom of the cell is made of a cover glass, allowing confocal microscopy observation through it. The particle cell containing the PMMA suspension is separated from the large reservoir containing AOT solutions by a semi-permeable membrane with a pore size of $0.22 \mu \mathrm{m}$. Ionic AOT micelles $(r \sim 1 \mathrm{~nm})$ can pass through the membrane, whereas PMMA particles $(\sigma \sim 1.8 \mu \mathrm{m})$ cannot. Thus, by adjusting the AOT concentration $C_{\mathrm{AOT}}$ in the reservoir, we can adjust the screening length of the repulsive Coulomb potential between particles in the sample cell through fast ion diffusion. We estimate the characteristic diffusion time $\tau$ of AOT micelles over a distance $h$ through the membrane to be $\tau \sim 10 \mathrm{~s}$, from $\tau \sim 6 \pi \eta h^{2} a / k_{\mathrm{B}} T$, where $\eta$ is the viscosity of the solvents $(1.3 \mathrm{cP}), a$ is the hydrodynamic radius of AOT micelles $(\sim 1 \mathrm{~nm})$ and $h$ is the depth of sample cells $(\sim 100 \mu \mathrm{m})$. We stress that this diffusion time is much shorter than the transition time, and thus, the substitution can be regarded as almost instantaneous. We have also confirmed that when the system is equilibrated after the substitution, there is no noticeable concentration gradient in the system. This conclusion is based on the experimental observations that there are no noticeable differences between the behaviours at the top and bottom of the sample cell. We have also estimated the flow rate from tracking the particle motion in a $2 \mathrm{D}$ horizontal plane to be less than $2 \mathrm{~nm} / \mathrm{s}$, indicating that our protocol is nonperturbative ${ }^{14}$. This special setup allows us to trigger and observe fcc-to-bcc martensitic transitions in situ at the single-particle level in real-time with 3D confocal microscopy.

Experimental protocol. We first prepare parent fcc crystals at a high $C_{\mathrm{AOT}}$. At $C_{\mathrm{AOT}} \sim 60 \mathrm{mM}(\phi \sim 22 \%)$, we can find a small amount of pre-existing bcc solid at the triple junction of grain boundaries, the sample walls, and inside the fcc grains. At $C_{\mathrm{AOT}} \sim 70 \mathrm{mM}(\phi \sim 22 \%)$, the pre-existing bcc becomes less frequent, and we can find defect-free fcc grains. Then, we replace the AOT solution in the reservoir by the low concentration one, indicated by the orange arrow in Fig. $1 \mathrm{~b}\left(C_{\mathrm{AOT}} \sim 10 \mathrm{mM}, \phi\right.$ $\sim 20 \%$, bcc-stable). It causes a quick ( $10 \mathrm{~s})$ reduction of the AOT concentration in the sample cell, triggering the fcc-to-bcc martensitic transition. We observe the transition process in-situ by a Leica SP8 fast confocal microscope $(\sim 10 \mu \mathrm{m} / \mathrm{s}$ along $z$ direction, with a $150 \times 150 \mu \mathrm{m}^{2} x-y$ view field). We recorded both thick regions (about $150 \mu \mathrm{m} \times 150 \mu \mathrm{m} \times 100 \mu \mathrm{m}$, including $\sim 250,000$ particles) with lower time resolution $(\sim 300 \mathrm{~s})$ and thin regions (about $150 \mu \mathrm{m} \times 150 \mu \mathrm{m} \times 20 \mu \mathrm{m}$, including $~$ 50,000 particles) with higher time resolution $(\sim 2.5 \mathrm{~s})$ for analysis. The field of view with the confocal microscope objective is about $150 \mu \mathrm{m} \times 150 \mu \mathrm{m}$, whereas the typical grain size in $(x, y)$ plane is about $100-300 \mu \mathrm{m}$. To avoid the bias of the sampling, we choose two working modes of a motorised scanning stage of our confocal microscope to make the large-field-of-view sampling: the large-filed-view modes (a $4 \times 4$ area that is much larger than the grain size) and the tile scanning mode (randomly pick up 16 regions). The confocal microscope can automatically do the $3 \mathrm{~d}$ scanning of each sub-region after we make the setup. The fractions of $70 \%$ and $30 \%$ indicated in the second column of Fig. le are estimated by the relative amounts of bcc crystallites formed through the GB-assisted pathway and in-grain nucleation, respectively, at the timing when the total bcc fraction is about $40-50 \%$. Because the final bcc crystallites formed through the two pathways will finally merge into large bcc grains (reaching nearly $90 \%$ ) under a high driving force, it is hard to discriminate their contributions from each other. On the other hand, the bcc crystals from the in-grain nucleation and GB-assisted nucleation can be distinguished in the growth stage when the total bcc fraction is about $40-50 \%$.

Structural analysis. We capture time-resolved 3D images and perform 3D particle tracking, using IDL 48 (http://www.physics.emory.edu/faculty/weeks//idl/index. html) and Trackpy ${ }^{49}$ (http://soft-matter.github.io/trackpy/v0.5.0/) to extract all particles' positions as a function of time, making the subsequent structural analysis possible. In this work, we use the two most frequently used structural order parameters, which are coarse-grained bond orientational order parameters, $W_{6}$ and $Q_{6}$ (see below for their definitions). $W_{6}$ is used to distinguish fcc and bcc by its sign ( $W_{6} \geq 0$ for bcc and $W_{6}<0$ for fcc), whereas $Q_{6}$ quantifies the degree of crystalline order of the solid (particles with $Q_{6}<0.35$ are regarded as defective structures, which mainly consist of highly distorted (defective) solid and a amall amount of liquid-like $\left(Q_{6}<0.25\right)$ particles. $)^{21,22,39-41}$ (Fig. 2).

Definition of order parameters, $\mathbf{Q}_{\mathbf{6}}, \mathbf{W}_{\mathbf{6}}$ and $\delta \boldsymbol{L}$. First, we calculate the local bond orientational order as $q_{l, m}(i)=\sum_{f \in F(i)} \frac{A(f)}{A} Y_{l, m}\left(\theta_{i, j}, \phi_{i, j}\right)$, where $A(f)$ is the surface area of the Voronoi cell facet $f$ separating centre particle $i$ and its neighbour $j$, and $A$ is the total surface area of the Voronoi cell boundary $F(i), Y_{l, m}\left(\theta_{i, j}, \phi_{i, j}\right)$ are the spherical harmonics with $m \in[-l, l], \theta_{i, j}$ and $\phi_{i, j}$ are the polar and azimuthal angles of the vector $\mathbf{r}_{i j}=\mathbf{r}_{i}-\mathbf{r}_{j}$, where $\mathbf{r}_{i}$ is the position vector of particle $i$ and $\mathbf{r}_{i}$ is the position vector of its neighbouring particle $j$. The coarse-graining version is calculated as $Q_{l, m}=\frac{1}{N b} \sum_{k=0}^{N b} q_{l, m}(k)$, with $N b$ being the number of neighbours determined by Voronoi cell analysis. Then, we obtain the rotationally invariant coarse-grained order parameters of particle $i, Q_{l}(i)$ and $W_{l}(i)$, as follows: $Q_{l}(i)=\left(\frac{4 \pi}{2 l+1} \sum_{m=-l}^{l}\left|Q_{l, m}(i)\right|^{2}\right)^{1 / 2}$ and $W_{l}(i)=\sum_{m_{1}+m_{2}+m_{3}=0}^{l}\left(\begin{array}{ccc}l & l & l \\ m_{1} & m_{2} & m_{3}\end{array}\right) \frac{Q_{l, m_{1}}(i) Q_{l, m_{2}}(i) Q_{l, m_{3}}(i)}{\left(\sum_{m=-l}^{l}\left|Q_{l m}(i)\right|^{2}\right)^{3 / 2}}$

We also introduce the Lindemann parameter $\delta L^{41}$. Here, $\delta L=\delta / a$, where $\delta^{2}$ is the height of the time-independent plateau of the mean-square displacement $\left\langle\Delta \mathbf{r}^{2}\right.$ $(t)\rangle$ and $a$ is the lattice constant. In this work, it is calculated from $-10 \mathrm{~s}$ to $+10 \mathrm{~s}$ for each particle.

Calculation of shear modulus $\mathbf{C}_{\mathbf{4 4}}$. The shear modulus is calculated, assuming the elastic interaction between particles and the Boltzmann distribution for the interparticle bond length ${ }^{41}$. We impose a shear matrix $\epsilon$ on bcc or fcc lattice matrix, then expand the strain energy $E$ into the combination of the spring constant $k$ and $\epsilon$. Then, $4 V_{0} C_{44}=\frac{d^{2} E}{d \epsilon^{2}}$. For bcc lattice, we have $C_{44}=\frac{k_{1}}{a}+\frac{2 k_{3}}{a}$. For fcc lattice, on the other hand, we have $C_{44}=\frac{k_{1}}{a}+\frac{2 k_{2} 50}{3 a}$. Here $a$ is the lattice constant, 
and $k_{1}, k_{2}$, and $k_{3}$ are the spring constants for nearest-neighbour, second-nearestneighbour and third-nearest-neighbour bonds, respectively. We determine $a$ from the $3 \mathrm{D}$ pair correlation functions. We measure each spring constant by determining the distribution of the corresponding bond length $l_{i}$, and assume that it obeys the equilibrium Boltzmann distribution as

$$
P\left(l_{i}\right)=A_{0} \exp ^{\frac{-E_{\text {elastic }}}{k_{\mathrm{B}} T}}=A_{0} \exp ^{\frac{k_{i}\left(x-l_{i}\right)^{2}}{2 k_{\mathrm{B}} T}}=A_{0} \exp ^{\frac{\left(x-A_{1}\right)^{2}}{2 A_{2}^{2}}} .
$$

We can experimentally get the neighbour bond length distribution at different time, and fit it with the last expression to get $A_{0}, A_{1}$, and $A_{2}$. Then, the elastic constant $k$ and average bond length $l$ are obtained as

$$
k_{i}=\frac{k_{\mathrm{B}} T}{A_{2}^{2}} \quad l=A_{1} .
$$

Shape-factor calculation using inertial tensor. For a quantitative description of bcc nuclei's shape, we take the following steps to define the shape-factor parameters using the inertial tensor. The inertial tensor of a cluster of particles with $N$ neighbours is defined as ${ }^{51}$ :

$$
\bar{I}=\sum_{i=1}^{N}\left(\vec{r}_{i} \cdot \vec{r}_{i}\right) \overline{1}-\vec{r}_{i} \bigotimes \vec{r}_{i}
$$

where $\vec{r}_{i}$ is the vector from the centre of mass of the cluster to particle $i, \overline{1}$ is the unit tensor, and $\otimes$ denotes tensor product. Then, we calculate the three eigenvalues of $\bar{I}, v_{1}, v_{2}$, and $v_{3}$, from the largest to smallest. Then, we define $S_{1}=v_{3} / v_{2}$ and $S_{2}=v_{3} / v_{1}$. Points on the $S_{1}-S_{2}$ space can characterise the cluster anisotropy.

\section{Data availability}

The experimental data that support the findings of this study are available within the article.

\section{Code availability}

The codes associated with this manuscript are available from the corresponding author upon request.

Received: 14 January 2021; Accepted: 8 June 2021;

Published online: 30 June 2021

\section{References}

1. Porter, D. A., Easterling, K. E. \& Sherif, M. Phase Transformations in Metals and Alloys (Revised Reprint) (CRC Press, 2009).

2. Kirby, S. H., Durham, W. B. \& Stern, L. A. Mantle phase changes and deepearthquake faulting in subducting lithosphere. Science 252, 216-225 (1991).

3. Smith, W. F. Principles of Materials Science and Engineering (McGraw Hill Series in Materials Science and Engineering) (McGraw-Hill College, 1995).

4. Nishiyama, Z. Martensitic Transformation (Academic Press, New York, 2012).

5. Sozinov, A., Likhachev, A. A., Lanska, N. \& Ullakko, K. Giant magnetic-fieldinduced strain in NiMnGa seven-layered martensitic phase. Appl. Phys. Lett. 80, 1746-1748 (2002).

6. Zahn, D. \& Leoni, S. Nucleation and growth in pressure-induced phase transitions from molecular dynamics simulations: Mechanism of the reconstructive transformation of $\mathrm{NaCl}$ to the CsCl-type structure. Phys. Rev. Lett. 92, 250201 (2004).

7. Delaey, L. Diffusionless Transformations, Phase Transformations in Materials (Wiley-VCH, 2001).

8. Koval, Y. N., Firstov, G. \& Odnosum, V. V. High temperature martensitic transformation and shape memory behavior in Hfir intermetallic compound. Mater. Sci. Forum 738-39, 72-76 (2013).

9. Teitelbaum, S. W. et al. Real-time observation of a coherent lattice transformation into a high-symmetry phase. Phys. Rev. X 8, 031081 (2018).

10. Chen, N.-K. et al. Directional forces by momentumless excitation and orderto-order transition in Peierls-distorted solids: the case of GeTe. Phys. Rev. Lett. 120, 185701 (2018).

11. Olson, G. B. \& Hartman, H. martensite and life : displacive transformations as biological processes. J. Phys. Colloq. 43, C4-855-C4-865 (1982).

12. Yethiraj, A., Wouterse, A., Groh, B. \& van Blaaderen, A. Nature of an electricfield-induced colloidal martensitic transition. Phys. Rev. Lett. 92, 058301 (2004).

13. Jenkins, I. C., Casey, M. T., McGinley, J. T., Crocker, J. C. \& Sinno, T. Hydrodynamics selects the pathway for displacive transformations in DNAlinked colloidal crystallites. Proc. Natl. Acad. Sci. USA 111, 4803-4808 (2014).

14. Peng, Y. et al. Diffusive and martensitic nucleation kinetics in solid-solid transitions of colloidal crystals. Nat. Commun. 8, 14978 (2017).
15. Chen, J., Weidner, D. J., Parise, J. B., Vaughan, M. T. \& Raterron, P. Observation of cation reordering during the Olivine-Spinel transition in Fayalite by in-situ synchrotron X-ray diffraction at high pressure and temperature. Phys. Rev. Lett. 86, 4072-4075 (2001).

16. Vives, E., Soto-Parra, D., Mañosa, L., Romero, R. \& Planes, A. Imaging the dynamics of martensitic transitions using acoustic emission. Phys. Rev. B 84, 060101 (2011)

17. Jacobs, K. Activation volumes for solid-solid transformations in nanocrystals. Science 293, 1803-1806 (2001).

18. Pogatscher, S., Leutenegger, D., Schawe, J., Uggowitzer, P. \& Löffler, J. Solid-solid phase transitions via melting in metals. Nat. Commun. 7, 11113 (2016).

19. Gasser, U. Real-space imaging of nucleation and growth in colloidal crystallization. Science 292, 258-262 (2001).

20. Tan, P., Xu, N. \& Xu, L. Visualizing kinetic pathways of homogeneous nucleation in colloidal crystallization. Nat. Phys. 10, 73-79 (2013).

21. Arai, S. \& Tanaka, H. Surface-assisted single-crystal formation of charged colloids. Nat. Phys. 13, 503-509 (2017).

22. Li, M., Chen, Y., Tanaka, H. \& Tan, P. Revealing roles of competing local structural orderings in crystallization of polymorphic systems. Sci. Adv. 6, eaaw8938 (2020).

23. Alsayed, A. M. Premelting at defects within bulk colloidal crystals. Science 309, 1207-1210 (2005)

24. Wang, Z., Wang, F., Peng, Y., Zheng, Z. \& Han, Y. Imaging the homogeneous nucleation during the melting of superheated colloidal crystals. Science 338, 87-90 (2012).

25. Weeks, E. R. Three-dimensional direct imaging of structural relaxation near the colloidal glass transition. Science 287, 627-631 (2000).

26. Mohanty, P. S., Bagheri, P., Nöjd, S., Yethiraj, A. \& Schurtenberger, P. Multiple path-dependent routes for phase-transition kinetics in thermoresponsive and field-responsive ultrasoft colloids. Phys. Rev. X 5 , 011030 (2015).

27. Du, C. X., van Anders, G., Newman, R. S. \& Glotzer, S. C. Shape-driven solid-solid transitions in colloids. Proc. Natl. Acad. Sci. USA 114, E3892-E3899 (2017).

28. Meijer, J.-M. et al. Observation of solid-solid transitions in $3 \mathrm{~d}$ crystals of colloidal superballs. Nat. Commun. 8, 14352 (2017).

29. Wan, D., Du, C. X., van Anders, G. \& Glotzer, S. C. FCC $\leftrightarrow$ BCC phase transitions in convex and concave hard particle systems. J. Phys. Chem. B 123, 9038-9043 (2019)

30. Weiss, J. A., Oxtoby, D. W., Grier, D. G. \& Murray, C. A. Martensitic transition in a confined colloidal suspension. J. Chem. Phys. 103, 1180-1190 (1995)

31. Peng, Y. et al. Two-step nucleation mechanism in solid-solid phase transitions. Nat. Mater. 14, 101-108 (2014).

32. Rossi, L. et al. Shape-sensitive crystallization in colloidal superball fluids. Proc Natl. Acad. Sci. USA 112, 5286-5290 (2015).

33. Besseling, T. H. et al. Oscillatory shear-induced $3 \mathrm{D}$ crystalline order in colloidal hard-sphere fluids. Soft Matter 8, 6931 (2012).

34. Ruiz-Franco, J. et al. Crystal-to-crystal transition of ultrasoft colloids under shear. Phys. Rev. Lett. 120, 078003 (2018).

35. Casey, M. T. et al. Driving diffusionless transformations in colloidal crystals using dna handshaking. Nat. Commun. 3, 1209 (2012).

36. Hsu, M. F., Dufresne, E. R. \& Weitz, D. A. Charge stabilization in nonpolar solvents. Langmuir 21, 4881-4887 (2005).

37. Kanai, T. et al. Crystallization and reentrant melting of charged colloids in nonpolar solvents. Phys. Rev. E 91, 030301 (2015).

38. Hwang, H., Weitz, D. A. \& Spaepen, F. Direct observation of crystallization and melting with colloids. Proc. Natl. Acad. Sci. USA 116, 1180-1184 (2019).

39. Steinhardt, P. J., Nelson, D. R. \& Ronchetti, M. Bond-orientational order in liquids and glasses. Phys. Rev. B 28, 784-805 (1983).

40. Mickel, W., Kapfer, S. C., Schröder-Turk, G. E. \& Mecke, K. Shortcomings of the bond orientational order parameters for the analysis of disordered particulate matter. J. Chem. Phys. 138, 044501 (2013).

41. Sprakel, J., Zaccone, A., Spaepen, F., Schall, P. \& Weitz, D. A. Direct observation of entropic stabilization of bcc crystals near melting. Phys. Rev. Lett. 118, 088003 (2017).

42. Gottstein, G., Molodov, D. A. \& Shvindlerman, L. S. Grain boundary migration in metals: recent developments. Interface Sci. 6, 7-22 (1998).

43. Hilgenkamp, H. \& Mannhart, J. Grain boundaries in high- $t_{c}$ superconductors. Rev. Mod. Phys. 74, 485-549 (2002)

44. Shan, Z. Grain boundary-mediated plasticity in nanocrystalline Nickel. Science 305, 654-657 (2004).

45. Loiseau, A. \& Vannuffel, C. TiAl 2 : a reentrant phase in the Ti-Al system. Phys Status Solidi A 107, 655-671 (1988).

46. Akahane, K., Russo, J. \& Tanaka, H. A possible four-phase coexistence in a single-component system. Nat. Commun. 7, 12599 (2016). 
47. Tsurusawa, H., Leocmach, M., Russo, J. \& Tanaka, H. Direct link between mechanical stability in gels and percolation of isostatic particles. Sci. Adv. $\mathbf{5}$, eaav6090 (2019).

48. Crocker, J. C. \& Grier, D. G. Methods of digital video microscopy for colloidal studies. J. Colloid Interface Sci. 179, 298-310 (1996).

49. Allan, D., Caswell, T., Keim, N. \& van der Wel, C. trackpy: Trackpy v0.3.1. http://soft-matter.github.io/trackpy/dev/introduction.html (2016).

50. Wallace, D. C. \& Callen, H. Thermodynamics of crystals. Amer. J. Phys. 40, 1718-1719 (1972).

51. Spellings, M. \& Glotzer, S. C. Machine learning for crystal identification and discovery. AIChE J. 64, 2198-2206 (2018).

\section{Acknowledgements}

We acknowledge financial support by the National Natural Science Foundation of China (Nos. 11774059, 11734014 and 12035004) and the Science and Technology Commission of Shanghai Municipality (No. 20JC1414700). H. Tanaka acknowledges Grants-in-Aid for Specially Promoted Research (JP25000002 and JP20H05619) and Scientific Research (A) (JP18H03675) from the Japan Society of the Promotion of Science (JSPS).

\section{Author contributions}

H. Tanaka and P.T. conceived and supervised the project. M.L., Z.Y. and Y.C. performed the experiments. M.L., Y.C., H. Tong, H. Tanaka and P.T. analysed the data, and M.L., H. Tanaka, and P.T. wrote the manuscript.

\section{Competing interests}

The authors declare no competing interests.

\section{Additional information}

Supplementary information The online version contains supplementary material available at https://doi.org/10.1038/s41467-021-24256-9.

Correspondence and requests for materials should be addressed to H.T. or P.T.

Peer review information Nature Communications thanks Andrei Petukhov and the other, anonymous, reviewer(s) for their contribution to the peer review of this work.

Reprints and permission information is available at http://www.nature.com/reprints

Publisher's note Springer Nature remains neutral with regard to jurisdictional claims in published maps and institutional affiliations.

(c) Open Access This article is licensed under a Creative Commons Attribution 4.0 International License, which permits use, sharing, adaptation, distribution and reproduction in any medium or format, as long as you give appropriate credit to the original author(s) and the source, provide a link to the Creative Commons license, and indicate if changes were made. The images or other third party material in this article are included in the article's Creative Commons license, unless indicated otherwise in a credit line to the material. If material is not included in the article's Creative Commons license and your intended use is not permitted by statutory regulation or exceeds the permitted use, you will need to obtain permission directly from the copyright holder. To view a copy of this license, visit http://creativecommons.org/ licenses/by/4.0/.

(C) The Author(s) 2021 